\title{
A STUDY OF THREE-NODE TRIANGULAR PLATE BENDING ELEMENTS
}

\author{
JEAN-LOUIS BATOZ \\ Departement de Génie Mécanique, Université de Technologie de Compiègne, Compiègne, France \\ KLAUS-JÜRGEN BATHE AND LEE-WING HO \\ Department of Mechanical Engineering, Massachusetts Institute of Technology, Cambridge, Massachusetts, U.S.A.
}

\begin{abstract}
SUMMARY
An assessment of flat triangular plate bending elements with displacement degrees-of-freedom at the three corner nodes only is presented, with the purpose of identifying the most effective for thin plate analysis. Based on a review of currently available elements, specific attention is given to the theoretical and numerical evaluation of three triangular 9 degrees-of-freedom elements; namely, a discrete Kirchhoff theory (DKT) element, a hybrid stress model (HSM) element and a selective reduced integration (SRI) element. New and efficient formulations of these elements are discussed in detail and the results of several example analyses are given. It is concluded that the most efficient and reliable three-node plate bending elements are the DKT and HSM elements.
\end{abstract}

\section{INTRODUCTION}

Since the earliest development of the finite element method, a considerable amount of research has been devoted to the analysis of plate and shell structures. A great number of papers have been published on this subject (see, for example, the review papers by Gallagher ${ }^{1,2}$ ). Considering the research and development efforts, various approaches, theories and variational principles to formulate plate and shell elements have been used to circumvent the difficulties that arise in the analysis of general plates and shells. However, despite the amount of research that has been devoted to the subject, further research effort is still required to provide the practising engineers with reliable, cost-effective and accurate computer programs ${ }^{3,4}$ to solve various plate assemblies and complex shell problems.

In essence, three approaches are being followed in the development of plate and shell elements:

1. A particular shell theory is used and discretized. ${ }^{1.5}$

2. Three-dimensional continuum equations are used and discretized (isoparametric elements) (References 6 and 7, etc.).

3. Plate bending and membrane element stiffnesses are superimposed and assembled in a global co-ordinate system (References 8 and 9, etc.).

The three approaches have advantages and disadvantages, and it is still difficult to state which of the three approaches is most effective based on criteria combining accuracy, computational cost and simplicity in use (in the data input phase as well as in the interpretation of results). Approach 3 received a great deal of attention for the linear analysis of shell structures in the mid-1960s, ${ }^{10}$ but the activities related to approaches 1 and 2 have dominated the past 10 years. It is only recently that a new impetus has been given to the analysis of shells using approach 3 . 
Considering this approach, triangular flat elements having displacements and rotations at the corner nodes as degrees-of-freedom-the engineering dof-are particularly appealing for many practical reasons; for example, arbitrary shell geometries, general supports and cut-outs, and beam stiffeners can be modelled. These elements have a total of 18 dof ( 3 translations and 3 rotations at each node) or 15 dof ( 3 translations and 2 rotations) depending on whether the rotation about the normal is included as a dof. The element formulation is based on a superposition of membrane and bending actions. Among the most recent papers on this subject, References 12 and 13 deal with the linear analysis, whereas References $9,14,8$ and 15 deal with the geometrically nonlinear analysis of shells with large displacements and rotations. In References 12,9 and 14 the hybrid stress formulations are used, whereas in References 13, 8 and 15, displacement-type formulations are employed.

A very important consideration in the development of these shell elements is the representation of the bending behaviour. Although several theoretical and numerical studies on plate bending finite elements have appeared in the past 15 years, a detailed recent study and comparison of triangular plate bending elements with only 3 dof at the corner nodes (displacement $w$ and rotations $\theta_{x}$ and $\theta_{y}$, as shown in Figure 1 ) is not available.

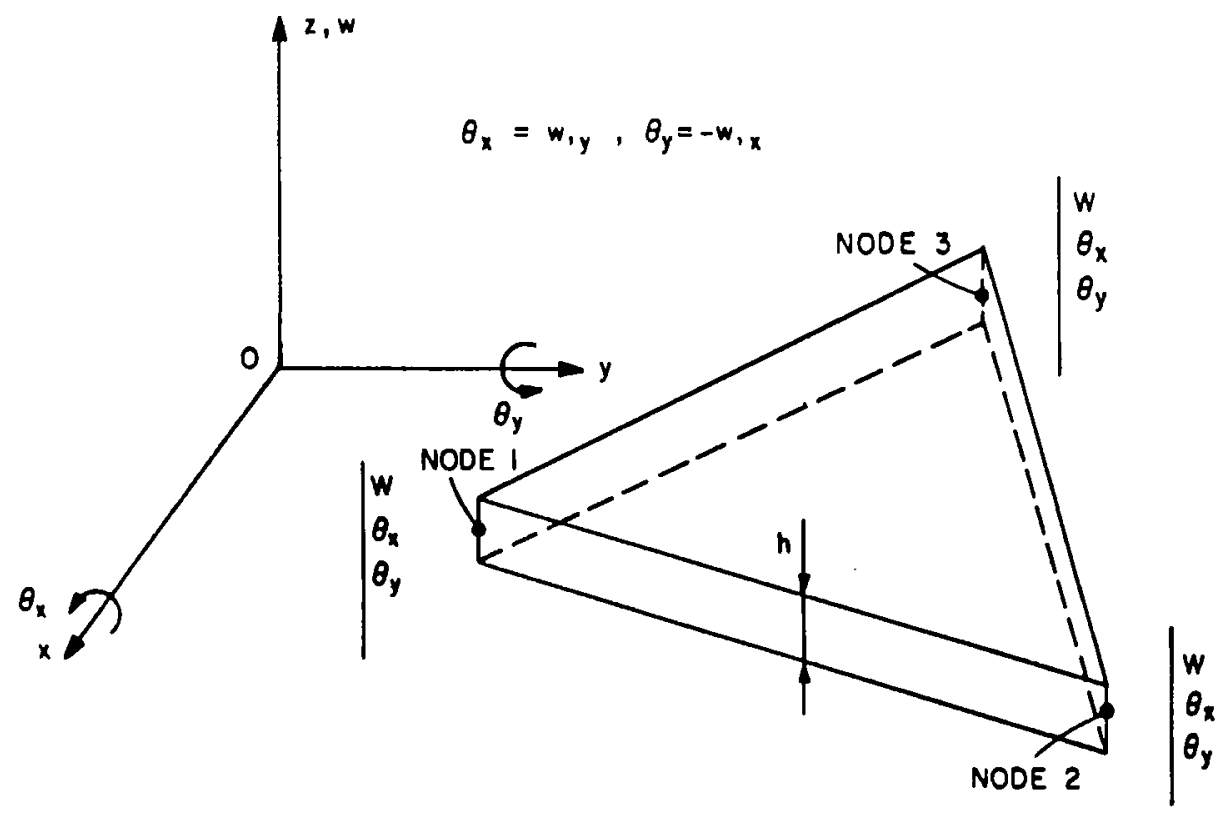

Figure 1. Nine dof triangular plate bending element

In this paper we present the results of a detailed theoretical and numerical study of triangular plate bending elements with 9 corner dof only. The objective in this study was to identify or develop an optimum element for the general linear analysis of plate bending problems. Our ultimate aim is to employ this element as the basis of a 3-noded shell element that can be used effectively for general geometric and material nonlinear analysis of thin shells. In our work specific attention was given to the soundness of the theoretical formulation, the numerical efficiency and reliability of the plate bending elements. It is apparent that very accurate and efficient elements can be developed for the analysis of specific problems only. However, unless 
an element always yields a stable solution, it cannot be recommended for use in a general analysis computer program, where reliability of analysis results is of utmost concern. These thoughts are particularly important in geometric nonlinear analysis in which the structural configuration changes continuously, and it is impractical and dangerous to apply 'rules' that tell under what boundary conditions and for which structural shapes an element can be employed.

In this paper we first review alternative formulations of 3-noded triangular plate bending elements that have been presented in the literature. This review suggests giving specific attention to the theoretical formulation and numerical evaluation of three elements: a DKT (discrete Kirchhoff theory) element, a HSM (hybrid stress model) element and a SRI (selective reduced integration) element. New and compact formulations of these elements are given in the paper, and to identify the relative effectiveness of the elements the results of various sample analyses using these elements are presented.

\section{REVIEW OF TRIANGULAR PLATE BENDING ELEMENTS WITH 9 DOF}

In this section we review the formulation and behaviour of the available triangular plate bending elements with 9 dof: the displacement $w$ and the rotations $\theta_{x}$ and $\theta_{y}$ at the three corner nodes. The converged solution is the classical Kirchhoff thin plate solution. These elements can be classified as

1. Displacement models based on the classical Kirchhoff thin plate theory.

2. Hybrid stress models based also on the Kirchhoff plate theory.

3. Displacement models derived from the theory of plates with transverse shear deformations.

\subsection{Displacement-based Kirchhoff plate theory elements}

The early attempts to develop satisfactory triangular elements with 9 dof are reported in References 16 and 17. The element formulations are based on the principle of minimum potential energy, where the compatibility requirements involve the displacements $w$ and the rotations $w_{, x}=-\theta_{y}$ and $w_{, y}=\theta_{x}$. Elements labelled $A, T$ and $T-10$ in Reference 16 are based on cubic polynomials in local co-ordinates $x$ and $y$. Their ineffectiveness is due to the following reasons: incompleteness $(A)$, incompatibility $(A, T, T-10)$, lack of invariance with regard to element orientation ( $T, A$ ) and singularity $(T)$. It was then realized that it is impossible to formulate a compatible triangular element with 9 dof with a single-field polynomial expansion

for $w$. One of the first compatible triangular elements is the well-known HCT element. ${ }^{16}$ Its formulation is based on the subdivision of the complete element into three subtriangles. An incomplete cubic (9-term) polynomial is used in each subregion for the displacement $w$, and the normal slope along the exterior edge of each region varies linearly. The formulation of this element has also been discussed using area co-ordinates ${ }^{18}$ (where it is called LCCT-9). The HCT element has frequently been regarded as a reference element for bending analysis of plates, mainly because of the extensive numerical results presented with its formulation. However, the formulation involves cumbersome algebraic manipulations and the element is rather stiff. The element has also been the subject of several theoretical studies (e.g. order of convergence, error estimates). ${ }^{19}$

In Reference 17 a non-conforming element (labelled BCIZ1 in this study) and a conforming element (labelled BCIZ2) are presented. The BCIZ1 element is a non-conforming element that satisfies the rigid body mode and the constant strain states. The shape functions expressed in area co-ordinates are given explicitly in some textbooks. ${ }^{10.20}$ The important drawback of the $\mathrm{BCIZ1}$ element is due to its incompatibility: it does not converge to the exact answer for some 
mesh patterns. The study of this drawback is given in Reference 17 and can be understood as the first application of what is now called the 'patch-test'.

The BCIZ2 element is a compatible element obtained by appropriate superposition of polynomials and rational shape functions in area co-ordinates. Due to the presence of these rational functions, a very high-order numerical integration scheme is needed (16 points in Reference 17) to evaluate the stiffness matrix. Some results for free vibrations of plates using the $\mathrm{BCIZ1}$ and $\mathrm{BCIZ2}$ elements are given in Reference 17, where better results are reported using the BCIZ1 element for the meshes and problems considered. The A-9 ${ }^{21}$ element is derived from the BCIZ2 element by replacing the true second derivatives of the shape functions with smoothed derivatives. These pseudo-derivatives are the least square linear polynomial versions of the true second derivatives, and the result is that only three numerical integration points are needed to evaluate the stiffness matrix. The pseudo-derivatives are not given explicitly in Reference 21, but good numerical results are reported for several static problems.

In Reference 21 a close relationship of the A-9 element to a hybrid stress formulation has been reported. At present the A-9 element replaces the BCIZ1 and BCIZ2 elements in some computer codes (Reference 3, Chapter 13).

In the computer program ICES-STRUDL II, the element CPT is a 9 dof triangular element. ${ }^{22}$ Two subtriangles are used in the formulation. The normal slope continuity is enforced along the three sides but the transverse displacement is not inter-element continuous along one side. ${ }^{23}$ Only a few numerical results using this element have been published.

\subsection{Hybrid stress elements}

The elements described above have been derived from the principle of minimum potential energy and the Kirchhoff plate theory. The hybrid stress (or hybrid mixed) method ${ }^{24}$ was developed to overcome the difficulties that are encountered in the development of pure displacement models due to element compatibility requirements. Various authors have presented hybrid stress triangular elements for plate bending problems. ${ }^{9}$ The most effective and also simplest element is called the HSM element in this study. This triangular bending element is derived from the Kirchhoff plate theory. The element has a linear distribution of bending moments in the interior and a cubic displacement variation with a linear normal slope $(w, n)$ variation along the edges of the element. The formulation of the HSM element has been discussed by several authors ${ }^{12,25-28}$ and slightly different ways have been devised to obtain the stiffness matrix depending on the expression of the functional used. The derivation of the stiffness matrices of hybrid stress elements appears to be rather cumbersome, and the evaluation of the element matrices appears to involve more algebraic manipulations and computer storage than comparative displacement models. However, some effective techniques are available to evaluate the stiffness matrix (References 9 and 26; see also Section 3.2).

Extensive numerical results are available with the HSM element for static as well as free vibration analyses of plates, and it is certainly one of the most effective 9 dof triangular elements available. Its formulation is given in detail in Section 3.2, where the stiffness matrix is given in compact form. Theoretical studies related to hybrid stress models and the HSM element are found in References 26,29 and 30.

In References 27 and 31 the HSM element has been derived using a hybrid displacement approach instead of the hybrid stress model. Hence, several alternatives are available to formulate the stiffness matrix of the HSM element; however, they do not seem to offer any advantage over the standard hybrid stress method as far as the derivation of the stiffness matrix is concerned. The main advantage of the hybrid displacement approach ${ }^{31}$ for deriving the HSM 
element lies in the more simple and accurate calculation of consistent loads due to pressure loading. Identical numerical results to the A-9 element ${ }^{21}$ are obtained. The calculations presented in Reference 21 require more basic operations than our evaluation presented in Section 3.2, but about the same high speed storage.

In Reference 51 a triangular hybrid stress element based on the theory of plates including transverse shear effects has been used to build quadrilateral elements with 12 dof. This element appears to be quite effective for plates of various length-to-thickness ratios and for sandwich plates.

Another class of 9 dof triangular elements for the analysis of Kirchhoff plates can be obtained with the so-called simplified hybrid displacement method (Reference 52 among others) by using a 10-term cubic polynomial for $w$ and correcting the stiffness matrix (which is the same as the $\mathrm{T}-10$ element in Reference 16$)$ to restore the continuity of $w, n$ along the sides. The resulting element is a 10 dof element; the reduction to 9 dof is carried out by static condensation. A critical assessment of this triangular element is given in Reference 32, where it is shown that the stiffness matrix can in some cases be singular (after the introduction of boundary conditions). Acceptable results are reported and applications to linear and nonlinear analysis of plates and shells have been given, but the elements are not reliable.

\subsection{Discrete Kirchhoff theory elements}

The formulation of elements based on the discrete Kirchhoff theory for bending of thin plates is obtained by first considering a theory of plates including transverse shear deformations (see Section 3.1). In this case the independent quantities are the deflection $w$, and the rotations $\beta_{x}$ and $\beta_{y}$ and only $C^{0}$ continuity requirements need to be satisfied. The transverse shear energy is neglected altogether and the Kirchhoff hypothesis is introduced in a discrete way along the edges of the element to relate the rotations to the transverse displacements. This approach has been used to formulate effective 9 dof triangular bending elements ${ }^{33-35}$ that converge to the classical thin plate solution. The element labelled QQ3 in Reference 33 and $\mathrm{KC}$ in Reference 36 were the most effective at the time of their presentation. Together with the HSM element they still represent the most effective 9 dof triangular plate bending elements available (QQ3 and KC are labelled DKT, discrete Kirchhoff theory, in our study). However, they are not well known, which can be attributed to several reasons. The formulation might appear to be 'strangelooking' (mathematically cloudy is the expression used in Reference 37) and the presentations used in References 33-36 are neither attractive nor simple. No special variational principle is associated with the model. Several plate and shell triangular elements based on the discrete Kirchhoff theory used subregions and/or mid-nodes ${ }^{33.34 .36}$ and the potential of the basic simple elements themselves was not recognized completely. Some researchers found the practical application of the element difficult, ${ }^{41}$ or the implementation complicated. ${ }^{42}$ Furthermore, it was claimed that the element predicts stresses relatively poorly. ${ }^{43}$ The final result is that the DKT element has not received widespread adoption and has also not been implemented in any major computer code.

In this paper we demonstrate the generally good behaviour of the DKT element in several analyses (Section 4). These results are also complemented by recent theoretical studies. ${ }^{19,38-40}$. In Reference 19, an approximation theory is used to derive error estimates associated with the DKT model. It is proved that the displacements and the free vibration eigenvalues are converging quadratically to the $C^{1}$ Kirchhoff solution of thin plates.

The above theoretical results are in agreement with the results obtained in References 38, 39 and 40 . In these papers the development of $C^{0}$ plate bending elements of quadratic accuracy is 
thoroughly described, both theoretically and numerically. In Reference 39 a family of triangular 9 dof plate bending elements with a parameter $c$ to control their fiexibility are presented. The formulation of these elements is similar to the formulation of the DKT element and the discrete Kirchhoff constraints are also introduced along the sides. The DKT element can be considered as the element of the family presented in Reference 39 with $c=0$. Imposing the Kirchhoff hypothesis along the sides of the elements and keeping the transverse shear energy is somehow contradictory; however, the converged solution is the classical thin plate solution and for different values of $c$ the convergence is quadratic ( $c=0$ included). Depending on the value of $c$, different results are obtained for coarse meshes. The choice of the optimum value of $c$ is therefore problem-dependent. However, the arguments and derivations given in the papers are valuable to justify the DKT element. The formulation of triangular elements for plates with $C^{0}$ deflection fields not described by the Kirchhoff bending theory is discussed in Reference 44 .

\subsection{Selective reduced integration element}

Recent and somehow successful developments of a beam element, quadrilateral plate elements $^{42,54}$ and axisymmetric shell elements, ${ }^{55}$ based on selective reduced integration concepts and the theory of plates including transverse shear effects, suggest that a simple selectively integrated triangular plate element with 9 dof may be effective. The formulation of such an element is presented in Section 3.3 and some numerical results using the element are summarized in Section 4.3. Based on these results it is concluded that the element is not effective when compared with the HSM and DKT elements.

\section{FORMULATION OF THE STIFFNESS MATRIX OF THE DISCRETE KIRCHHOFF THEORY ELEMENT AND THE HYBRID STRESS MODEL ELEMENT}

\subsection{This discrete Kirchhoff theory element (DKT)}

Before presenting the derivation of the DKT element, we briefly summarize the theory of plates including transverse shear that is used in the element derivation.

3.1.1. Small displacement theory of plates with transverse shear included. The theory of plates with transverse shear deformations included (the plate theory of Reissner or Mindlin ${ }^{46}$ ) uses a generalization of the Kirchhoff hypothesis: "points of the plate originally on the normal to the undeformed middle surface remain on a straight line but which is not necessarily normal to the deformed middle surface.' With this assumption in the small displacement bending theory the displacement components of a point of co-ordinates $x, y, z$ are:

$$
u=z \beta_{x}(x, y) ; \quad v=z \beta_{y}(x, y) ; \quad \text { and } w=w(x, y)
$$

where $w$ is the transversal displacement, $\beta_{x}$ and $\beta_{y}$ are the rotations of the normal to the undeformed middle surface in the $x-z$ and $y-z$ planes, respectively, Figure 2. (In the Kirchhoff plate theory, $\beta_{x}=-w_{, x}$ and $\beta_{y}=-w, y$.)

The linear strain expressions are

(a) The bending strains (linear through the thickness)

$$
\varepsilon_{b}=z K
$$



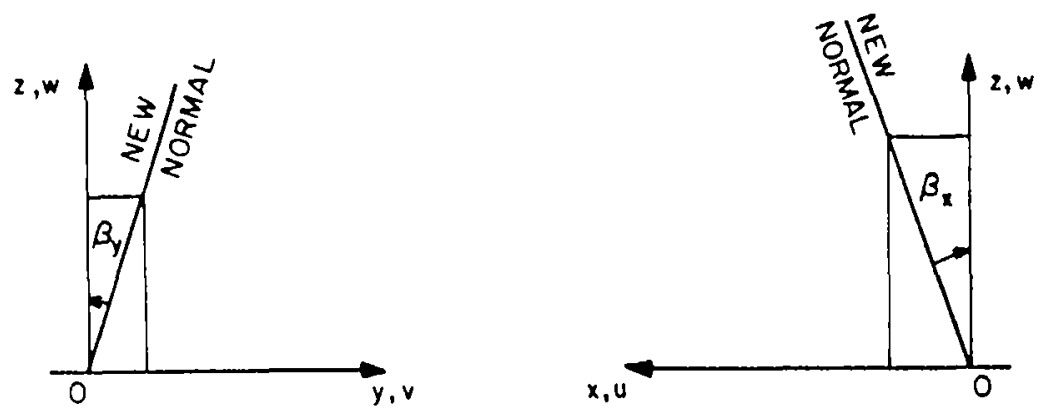

Figure 2. Positive directions of $\beta_{x}$ and $\beta_{y}$

where $k$ is the three-component vector of curvatures:

$$
\kappa=\left[\begin{array}{c}
\beta_{x, x} \\
\beta_{y, y} \\
\beta_{x, y}+\beta_{y, x}
\end{array}\right]
$$

(b) The transverse shear strains (constant through the thickness)

$$
\gamma=\left[\begin{array}{l}
w, x \\
w_{, y}+\beta_{x}
\end{array}\right]
$$

As in the Kirchhoff plate theory, the state of stress in the plate is defined with plane stress assumption, i.e. $\sigma_{z}=0$, and with the assumption of plane anisotropy (no coupling between $\sigma_{b}$ and $\gamma$ ). In the most general case of multilayered composite plates and sandwich plates, the stress-strain relations (for an elastic material) for a particular layer are:

$$
\boldsymbol{\sigma}_{b}=\left[\begin{array}{c}
\sigma_{x} \\
\sigma_{y} \\
\tau_{x y}
\end{array}\right]=z\left[\begin{array}{lll}
D_{11} & D_{12} & D_{13} \\
& D_{22} & D_{23} \\
(s y m) & D_{33}
\end{array}\right] \quad K=z D_{K}
$$

and

$$
\boldsymbol{\sigma}_{\mathrm{s}}=\left[\begin{array}{c}
\tau_{x z} \\
\tau_{y z}
\end{array}\right]=\left[\begin{array}{ll}
E_{13} & E_{23} \\
E_{23} & E_{33}
\end{array}\right] \quad \boldsymbol{\gamma}=\mathbf{E} \boldsymbol{\gamma}
$$

where the $E_{i j}, i, j=1,3$, are the components of the three-dimensional elasticity matrix and

$$
D_{i j}=E_{i j}-\frac{E_{i 3} E_{3 i}}{E_{33}}
$$

With the kinematics as given by equations (1)-(4) and the material description as given by equations (5) and (6) the strain energy is

$$
U=U_{b}+U_{s}
$$

where

$$
\begin{aligned}
& U_{b}=\frac{1}{2} \int_{A} \boldsymbol{\kappa}^{\mathrm{T}} \mathbf{D}_{b} \boldsymbol{\kappa} \mathrm{d} x \mathrm{~d} y \\
& U_{s}=\frac{1}{2} \int_{A} \boldsymbol{\gamma}^{\mathrm{T}} \mathbf{D}_{s} \boldsymbol{\gamma} \mathrm{d} x \mathrm{~d} y
\end{aligned}
$$


and

$$
\mathbf{D}_{b}=\int_{-h / 2}^{h / 2} \mathbf{D}(z) z^{2} \mathrm{~d} z ; \quad \mathbf{D}_{s}=\mathbf{k} \int_{-h / 2}^{h / 2} \mathbf{E}(z) \mathrm{d} z \mathbf{k}
$$

The variables $U_{b}$ and $U_{s}$ represent the bending and transverse shear contributions, respectively, and $\mathbf{k}$ in equation (11) contains shear correction factors to account for the non-uniformity of the transverse shear stresses through the plate thickness.

In equations (8) and (9) the matrices $D_{b}$ and $D_{s}$ are functions of the thickness of the plate, $h$, and of the elastic properties of the different layers; the variable $A$ is the area of the middle surface of the plate. For the important practical case of an isotropic homogeneous plate of constant thickness we have

$$
\mathbf{D}_{b}=\frac{E h^{3}}{12\left(1-\nu^{2}\right)}\left[\begin{array}{ccc}
1 & \nu & 0 \\
& 1 & 0 \\
(\mathrm{sym}) & & \frac{1-\nu}{2}
\end{array}\right] ; \quad \mathbf{D}_{s}=\frac{E h k}{2(1+\nu)}\left[\begin{array}{ll}
1 & 0 \\
0 & 1
\end{array}\right]
$$

The explicit expressions $U_{b}$ and $U_{s}$ are then

$$
\begin{gathered}
U_{b}=\frac{E h^{3}}{24\left(1-\nu^{2}\right)} \int_{A}\left\{\beta_{x, x}^{2}+\beta_{y, y}^{2}+2 \nu \beta_{y, y} \beta_{x, x}+\frac{1-\nu}{2}\left(\beta_{y, x}+\beta_{x, y}\right)^{2}\right\} \mathrm{d} x \mathrm{~d} y \\
U_{s}=\frac{E h k}{4(1+\nu)} \int_{A}\left\{\left(w, x+\beta_{x}\right)^{2}+\left(w, y+\beta_{y}\right)^{2}\right\} \mathrm{d} x \mathrm{~d} y
\end{gathered}
$$

The variables $E$ and $\nu$ in equations (12) and (14) are the Young's modulus and Poisson's ratio, and $k$ is the shear correction factor usually taken as $5 / 6$.

By definition, the bending moments $\mathbf{M}$ and shear forces $\mathbf{Q}$ are obtained by integration of the stresses through the thickness:

$$
\mathbf{M}=\left[\begin{array}{c}
M_{x} \\
M_{y} \\
M_{x y}
\end{array}\right]=\int_{-h / 2}^{h / 2} \sigma z \mathrm{~d} z=\mathbf{D}_{b} \boldsymbol{K}
$$

and

$$
\mathbf{Q}=\left[\begin{array}{l}
Q_{x} \\
Q_{y}
\end{array}\right]=\mathbf{k} \int_{-h / 2}^{h / 2} \sigma_{s} d z k=D_{s} \gamma
$$

The expression of $U$ as given by equations (7)-(9), or (13) and (14), is used to formulate finite elements for the analysis of thick plates, moderately thick plates, sandwich plates and multilayered plates where the transverse shear effects are important. The independent quantities subjected to variation are $w, \beta_{x}$ and $\beta_{y}$ with the conditions that $w=\bar{w}, \beta_{x}=\bar{\beta}_{x}$ and $\beta_{y}=\bar{\beta}_{y}$ on the part of the boundary where displacements are prescribed. The $C^{0}$ continuity requirements are not difficult to fulfil and various elements can be developed. In thin plate analysis, selective reduced integration has been employed for low-order elements ${ }^{42,47}$ (see Section 3.3). It is interesting to note that with high-order elements neither reduced nor selective integration is required. ${ }^{6,17}$

3.1.2. Stiffness matrix of the DKT element. For thin plates the transverse shear strains and therefore the transverse shear strain energy $U_{s}$ are negligible compared to the bending energy. 
The finite element model based on the functional in equations (7)-(9), or (13) and (14), must be able to represent this constraint. Therefore, the stiffness matrix of the DKT element for the analysis of thin plates is based only on the expression

$$
U=\frac{1}{2} \int_{A} \boldsymbol{K}^{\mathrm{T}} \mathbf{D}_{b} \mathbf{K} \mathrm{d} x \mathrm{~d} \boldsymbol{y}
$$

where $A$ is the middle surface of an element and $K$ and $D_{b}$ are given by (3) and (10), or (12a), respectively.

Equation (17) contains only the first derivatives of $\beta_{x}$ and $\beta_{y}$ and hence it is relatively easy to establish interpolation functions that satisfy the compatibility requirements. However, since $\beta_{x}$, $\beta_{y}$ are the variables in equation (17), it is necessary to relate the rotations of the normal to the middle surface to the transverse displacement $w$ (which does not appear in equation (17)). This goal should be achieved with the following considerations:

(a) The triangular element must have only 9 dof; that is, the displacement $w$ and the rotations $\theta_{x}$ and $\theta_{y}$ at the three corner nodes.

(b) Since the Kirchhoff solution of thin plates is sought, the nodal point rotations should be $\theta_{x}=+w, y$ and $\theta_{y}=-w, x$ (so that the Kirchhoff kinematical boundary conditions be satisfied).

(c) Since the element models thin plates, as governed by the Kirchhoff plate theory, the Kirchhoff plate theory assumptions can be imposed at any discrete points.

(d) The compatibility of the rotations $\beta_{x}$ and $\beta_{y}$ should not be lost.

The formulation of the DKT element as presented in References (33)-(36) is based on the following assumptions:

(1) $\beta_{x}$ and $\beta_{y}$ vary quadratically over the element, i.e.

$$
\beta_{x}=\sum_{i=1}^{6} N_{i} \beta_{x_{i}} ; \quad \beta_{y}=\sum_{i=1}^{6} N_{i} \beta_{y_{i}}
$$

where $\beta_{x_{i}}$ and $\beta_{y_{i}}$ are the nodal values at the corners and at the mid-nodes, Figure 3 , the $N_{i}(\xi, \eta)$ are the shape functions given in Appendix A, $\xi$ and $\eta$ are the area co-ordinates;

(2) The Kirchhoff hypothesis is imposed at:

(a) the corner nodes

$$
\gamma=\left[\begin{array}{c}
\beta_{x}+w, x \\
\beta_{y}+w, y
\end{array}\right]=0 \quad \text { at nodes } 1,2 \text { and } 3
$$

(b) the mid-nodes (defined anticlockwise around the element boundary, Figure 3) i.e.

$$
\beta_{s_{k}}+w_{s_{k}}=0 \quad k=4,5,6
$$

(3) The variation of $w$ along the sides is cubic, i.e.,

$$
w_{, s_{k}}=-\frac{3}{2 l_{i j}} w_{i}-\frac{1}{4} w_{s_{i}}+\frac{3}{2 l_{i j}} w_{j}-\frac{1}{4} w_{, s_{i}}
$$

with $k$ denoting the mid-node of side $i j$ and $l_{i j}$ equal to the length of the side $i j$.

(4) A linear variation of $\beta_{n}$ is imposed along the sides, i.e.

$$
\beta_{n_{k}}=\frac{1}{2}\left(\beta_{n_{i}}+\beta_{n_{j}}\right)
$$

where $k=4,5,6$ denotes the mid-node of the sides 23,31 and 12 , respectively. 


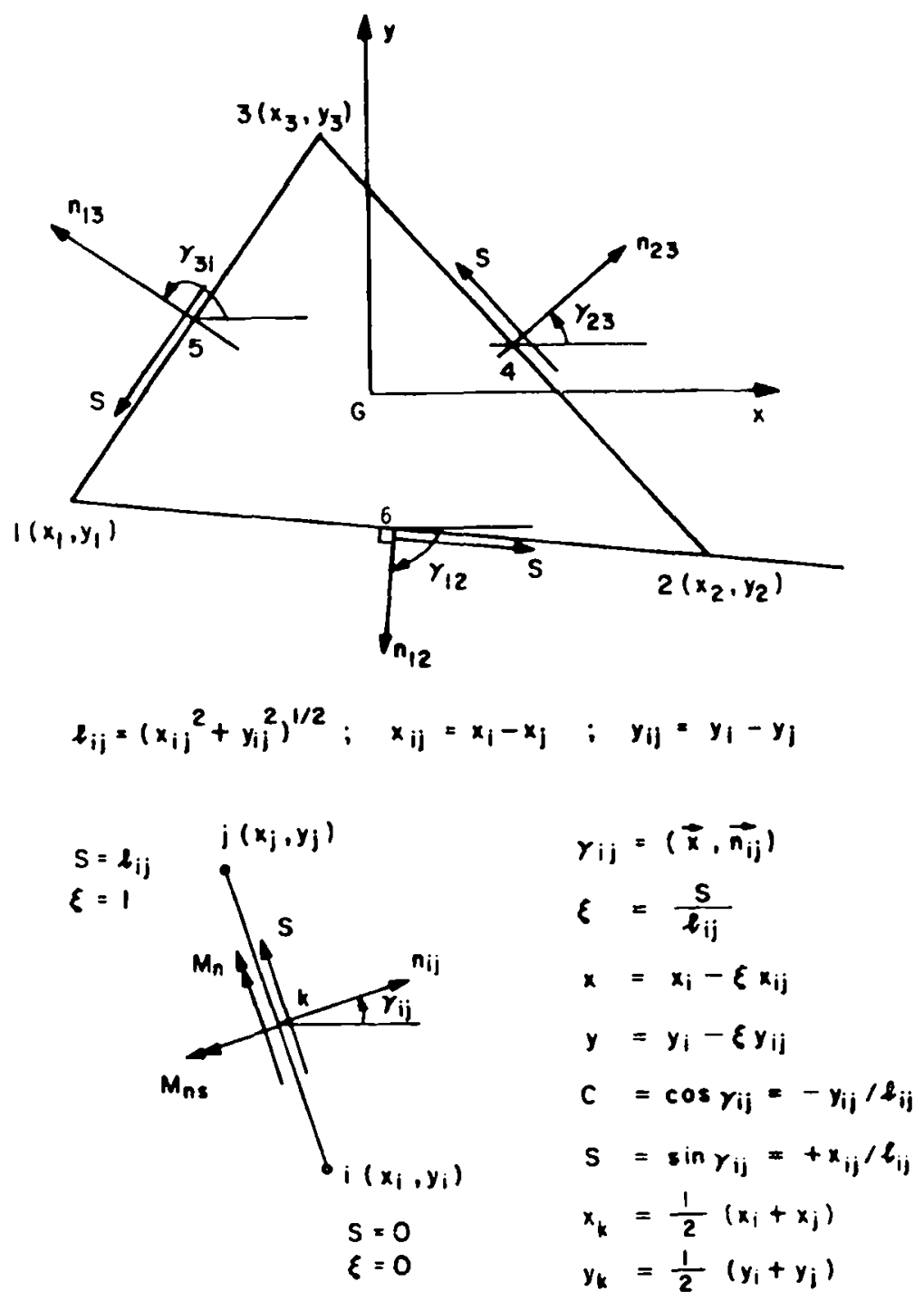

Figure 3. Geometry of the triangular element

It is appropriate here to make the following remarks before continuing with the derivation of the stiffness matrix.

(1) From the relations in equations (18)-(22) it is seer. that the relation between the rotations and the transverse displacement $w$ is given by equation (20) by assuming a cubic variation of $w$ along the sides (or a quadratic variation of $w, s$ ).

(2) There is no need to define an interpolation function for $w$ on the element (but the assumption of a cubic variation of $w$ along the sides is the property of a cubic polynomial on the element).

(3) Since $w$ varies cubically along the sides, $w$, varies quadratically and so does $\beta_{s}$. Since $w, s$ matches $\beta_{s}$ at the three points along each side, the Kirchhoff hypothesis $\left(\gamma_{s}=\beta_{s}+w_{, s}=0\right)$ is satisfied along the entire boundary $\partial \mathrm{A}$ (Figure 4 ). 


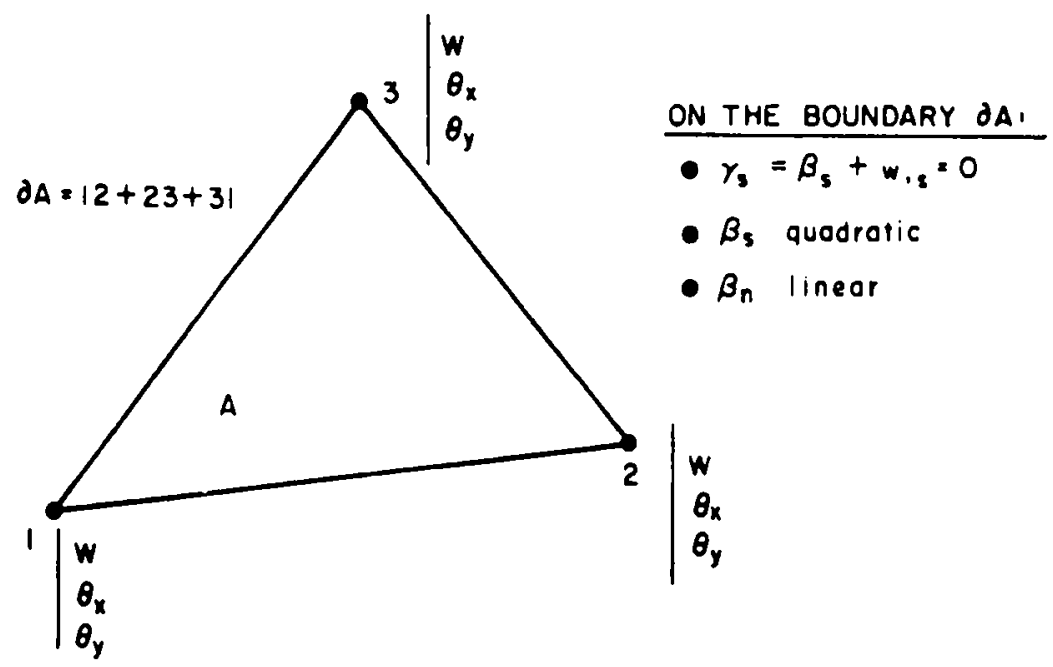

Figure 4. The DKT element

(4) Convergence towards the classical thin plate solution is obtained because the transverse shear strain energy is neglected, equation (17), and because the Kirchhoff hypothesis is satisfied along the element boundary.

(5) It follows from equations (8-22) that $w, w, s, \beta_{s}$ and $\beta_{n}$ are compatible (interelement continuous) along the sides (note that $w, n$ has not been introduced since $w$ is not defined in the interior).

(6) With the restriction imposed on $\beta_{n}$ in equation (22), $\beta_{x}$ and $\beta_{y}$ are given by complete polynomials of degree one in the element. The global discretization error in the energy is $0\left(l^{2}\right)$, where $l$ is the measure of the size of the element. ${ }^{19,39}$

(7) When the formulation is applied to a one-dimensional beam, the exact stiffness matrix of a thin beam (with a cubic polynomial $w$ ) is obtained.

(8) The formulation is readily extendable to obtain quadrilateral elements with 12 dof (e.g. the QC element in Reference 36) and other polygonal elements.

To obtain $\beta_{x}$ and $\beta_{y}$ in terms of the nodal dof

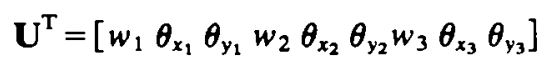

the following geometrical relations are needed on each side:

$$
\left[\begin{array}{l}
\beta_{x} \\
\beta_{y}
\end{array}\right]=\left[\begin{array}{rr}
c & -s \\
s & c
\end{array}\right]\left[\begin{array}{l}
\beta_{n} \\
\beta_{s}
\end{array}\right]
$$

and

$$
\left[\begin{array}{l}
w, s \\
w_{, n}
\end{array}\right]=\left[\begin{array}{rr}
c & s \\
s & -c
\end{array}\right]\left[\begin{array}{l}
\theta_{x} \\
\theta_{y}
\end{array}\right]
$$

where $c=\cos \left(\vec{x}, \vec{n}_{i j}\right)$ and $s=\sin \left(\vec{x}, \vec{n}_{i j}\right)$, Figure 3 .

Using equations (18)-(25), the following expressions are obtained for $\beta_{x}$ and $\beta_{y}$ :

$$
\begin{aligned}
& \boldsymbol{\beta}_{x}=\mathbf{H}_{x}^{\mathbf{T}}(\xi, \eta) \mathbf{U} \\
& \beta_{y}=\mathbf{H}_{y}^{\mathbf{T}}(\xi, \eta) \mathbf{U}
\end{aligned}
$$


where $\mathbf{H}_{x}$ and $\mathbf{H}_{y}$ are the nine component vectors of new shape functions. The components are functions of the $N_{i}, i=1,6$ and the co-ordinates of the nodes,

$$
\begin{aligned}
& H_{x_{1}}=1.5\left(a_{6} N_{6}-a_{5} N_{5}\right) \\
& H_{x_{2}}=b_{5} N_{5}+b_{6} N_{6} \\
& H_{x_{3}}=N_{1}-c_{5} N_{5}-c_{6} N_{6} \\
& H_{y_{1}}=1.5\left(d_{6} N_{6}-d_{5} N_{5}\right) \\
& H_{y_{2}}=-N_{1}+e_{5} N_{5}+e_{6} N_{6} \\
& H_{y_{3}}=-H_{x_{2}}
\end{aligned}
$$

The functions $H_{x_{4}}, H_{x_{5}}, H_{x_{6}}, H_{y_{4}}, H_{y_{5}}$ and $H_{y_{6}}$ are obtained from the above expressions by replacing $N_{1}$ by $N_{2}$ and indices 6 and 5 by 4 and 6 , respectively. The functions $H_{x_{7}}, H_{x_{8}}, H_{x_{9}}, H_{y_{7}}$, $H_{y_{8}}$ and $H_{y_{9}}$ are obtained by replacing $N_{1}$ by $N_{3}$ and indices 6 and 5 by 5 and 4 , respectively. Also,

$$
\begin{aligned}
a_{k} & =-x_{i j} / l_{i j}{ }^{2} \\
b_{k} & =\frac{3}{4} x_{i j} y_{i j} / l_{i j}{ }^{2} \\
c_{k} & =\left(\frac{1}{4} x_{i j}{ }^{2}-\frac{1}{2} y_{i j}{ }^{2}\right) / l_{i j}{ }^{2} \\
d_{k} & =-y_{i j} / l_{i j}{ }^{2} \\
e_{k} & =\left(\frac{1}{4} y_{i j}{ }^{2}-\frac{1}{2} x_{i j}{ }^{2}\right) / l_{i j}{ }^{2} \\
l_{i j}{ }^{2} & =\left(x_{i j}{ }^{2}+y_{i j}{ }^{2}\right)
\end{aligned}
$$

where $k=4,5,6$ for the sides $i j=23,31,12$ respectively (Figure 3 ).

The evaluation of the stiffness matrix follows the standard procedures of the finite element displacement method.

Using equations (3) and (26),

$$
\mathbf{K}=\mathbf{B U}
$$

where $\mathbf{B}$ is the strain-displacement transformation matrix:

$$
\mathbf{B}(\xi, \eta)=\frac{1}{2 \mathrm{~A}}\left[\begin{array}{c}
y_{31} \mathbf{H}_{x, \xi}^{\mathrm{T}}+y_{12} \mathbf{H}_{x, \eta}^{\mathrm{T}} \\
-x_{31} \mathbf{H}_{y, \xi}^{\mathrm{T}}-x_{12} \mathbf{H}_{y, \eta}^{\mathrm{T}} \\
-x_{31} \mathbf{H}_{x, \xi}^{\mathrm{T}}-x_{12} \mathbf{H}_{x, \eta}^{\mathrm{T}}+y_{31} \mathbf{H}_{y, \xi}^{\mathrm{T}}+y_{12} \mathbf{H}_{y, \eta}^{\mathrm{T}}
\end{array}\right]
$$

and $2 \mathrm{~A}=x_{31} y_{12}-x_{12} y_{31}$.

The derivatives of $H_{x}$ and $H_{y}$ with respect to $\xi$ and $\eta$ are given explicitly in Appendix A.

The stiffness matrix of the DKT element becomes

$$
\mathbf{K}_{\mathrm{DKT}}=2 \mathrm{~A} \int_{0}^{1} \int_{0}^{1-\eta} \mathbf{B}^{\mathrm{T}} \mathbf{D}_{b} \mathbf{B} \mathrm{d} \xi \mathrm{d} \eta
$$

If the thickness and the material properties are constant over the element, the exact integration of $\mathbf{K}$ is obtained using three numerical integration points located at the mid-nodes or inside ${ }^{10}$ because the integral involves only quadratic terms. 
Once the nodal displacements have been calculated, the bending moments $M$ at any point in the element can be obtained using equations (15) and (29):

$$
\mathbf{M}(x, y)=\mathbf{D}_{b} \mathbf{B}(x, y) \boldsymbol{U}
$$

where

$$
\begin{aligned}
& x=x_{1}+\xi x_{21}+\eta x_{31} \\
& y=y_{1}+\xi y_{21}+\eta y_{31}
\end{aligned}
$$

Since, in general, $\mathbf{M}$ depends upon all the components of $\mathbf{U}, \mathbf{M}$ is not unique along the boundary shared by two elements.

\subsection{The hybrid stress model element (HSM)}

In the formulation of the HSM element the classical Kirchhoff thin plate theory is used directly. With the notation used in Section 3.1, $\gamma=0$ everywhere in the plate, $\beta_{x}=-w_{, x}=\theta_{y}$ and $\beta_{y}=-w_{, y}=-\theta_{x}$.

The so-called hybrid stress functional for plate bending under the Kirchhoff assumptions can be established: $24,30,46$

(a) either from the Hellinger-Reissner functional by assuming that the equilibrium equation of the plate in terms of bending moments is satisfied and that the geometrical boundary conditions are also satisfied, or

(b) from the complementary potential energy by relaxing the natural boundary conditions and by assuming that the geometrical boundary conditions are satisfied.

The following expression has been found convenient for the derivation of the stiffness matrix of the HSM element:

$$
U_{\mathrm{HS}}=\int_{A}-B\left(M_{i j}\right) \mathrm{d} x \mathrm{~d} y+\int_{\partial A}\left(w Q_{n}-w,{ }_{n} M_{n}-w, g M_{n s}\right) \mathrm{dS}
$$

where $A$ represents the middle surface of the element, $\partial A$ its boundary, and $B\left(M_{i j}\right)$ is the complementary energy per unit middle surface. For a general multilayered rigidly bonded composite plate:

$$
B\left(M_{i j}\right)=\frac{1}{2} \mathbf{M}^{\mathrm{T}} \mathbf{D}_{b}^{-1} \mathbf{M}
$$

where $M$ is the vector containing the bending moments $M_{x}, M_{y}, M_{x y}$, and $D_{b}$ is defined in equation (10). For the important practical case of a homogeneous isotropic plate, $\mathbf{D}_{b}$ is given in equation (12a) and

$$
B\left(M_{i j}\right)=\frac{12}{E h^{3}}\left\{\left(M_{x}+M_{y}\right)^{2}+2(1+\nu)\left(M_{x y}^{2}-M_{x} M_{y}\right)\right\}
$$

Also, $Q_{n}, M_{n}$ and $M_{n s}$ are the shear force, normal and twisting bending moments, respectively, along the boundary (Figure 3):

$$
\begin{aligned}
Q_{n} & =\mathrm{c}\left(M_{x, x}+M_{x y, y}\right)+\mathrm{s}\left(M_{y, y}+M_{x y, x}\right) \\
M_{n} & =\mathrm{cc} M_{x}+2 \mathrm{cs} M_{x y}+\mathrm{ss} M_{y} \\
M_{n s} & =-s c M_{x}+(\mathrm{cc}-\mathrm{ss}) M_{x y}+\mathrm{sc} M_{y}
\end{aligned}
$$


where $\mathrm{c}$ and $\mathrm{s}$ abbreviate $\cos (\vec{x}, \vec{n})$ and $\sin (\vec{x}, \vec{n})$, respectively; $\vec{n}$ is the (outward) normal along the contour.

The independent quantities subject to variation in the hybrid stress functional are the components, $M_{x}, M_{y}, M_{x y}$ in $A$ and $w$ and $w_{, n}$ along the contour $\partial A$ with the subsidiary conditions:

$$
M_{x, x x}+2 M_{x y, x y}+M_{y, y y}=0 \text { in } A
$$

and

$$
w=\bar{w}, w_{, n}=\bar{w}_{, n} \quad \text { on } C_{u}
$$

( $C_{u}$ is the part of the boundary on which displacements are prescribed.) It is assumed that uniform loading is represented by direct lumped loads.

The HSM element proposed by several authors (see Section 2 ) is based on the assumption that the bending moments vary linearly in the interior of the element and $w$ varies cubically along the sides with a linear variation of $w, n$. The nodal dof are the same as for the DKT element, equation (23). Thus, it is assumed that

$$
\mathbf{M}=\mathbf{P} \boldsymbol{\beta}
$$

where

$$
\begin{gathered}
\mathbf{P}=\left[\begin{array}{ccc}
\mathbf{P}_{0} & \mathbf{0} & \mathbf{0} \\
\mathbf{0} & \mathbf{P}_{0} & \mathbf{0} \\
\mathbf{0} & \mathbf{0} & \mathbf{P}_{0}
\end{array}\right] \\
\mathbf{P}_{0}=\left[\begin{array}{lll}
1 & x & y
\end{array}\right]
\end{gathered}
$$

$\beta$ is a nine-component vector of generalized parameters.

Using equations (35) and (40), the complementary strain energy is

$$
U_{1}=-\frac{1}{2} \boldsymbol{\beta}^{\mathrm{T}} \mathbf{H} \boldsymbol{\beta}
$$

where

$$
\mathbf{H}=\int_{A} \mathbf{P}^{\mathrm{T}} \mathbf{D}_{b}^{-1} \mathbf{P} \mathrm{d} x d y
$$

Owing to the nature of $\mathbf{P}$, the $9 \times 9$ matrix $\mathbf{H}$ can be expressed as

$$
\mathbf{H}=\left[\begin{array}{lll}
c_{11} \phi & c_{12} \phi & c_{13} \phi \\
c_{12} \phi & c_{22} \phi & c_{23} \phi \\
c_{13} \phi & c_{23} \phi & c_{33} \phi
\end{array}\right]
$$

where the $C_{i j}$ are the coefficients of the $\mathbf{D}_{b}^{-1}$ matrix and

$$
\boldsymbol{\phi}=\int_{A} \mathbf{P}_{0}^{\mathrm{T}} \mathbf{P}_{0} \mathrm{~d} x \mathrm{~d} y=\int_{A}\left[\begin{array}{lll}
1 & x & y \\
x & x^{2} & x y \\
y & x y & y^{2}
\end{array}\right] \mathrm{d} x \mathrm{~d} y
$$

If, for simplicity, the origin of the $x$ and $y$ local axes is taken at the element centroid (Figure 3 ), we have

$$
\phi=\left[\begin{array}{lll}
A & 0 & 0 \\
0 & \alpha & \psi \\
0 & \psi & \gamma
\end{array}\right]
$$


where $A$ is the surface area of the element,

$$
\begin{aligned}
\alpha & =\frac{A}{12}\left(x_{1}^{2}+x_{2}^{2}+x_{3}^{2}\right) \\
\psi & =\frac{A}{12}\left(x_{1} y_{1}+x_{2} y_{2}+x_{3} y_{3}\right) \\
\gamma & =\frac{A}{12}\left(y_{1}^{2}+y_{2}^{2}+y_{3}^{2}\right)
\end{aligned}
$$

( $x_{i}, y_{i} i=1,3$ are the co-ordinates of the nodes with the centroid as the origin.)

For the evaluation of the stiffness matrix we need to compute $\mathbf{H}^{-1}$ :

$$
\mathbf{H}^{-1}=\left[\begin{array}{lll}
d_{11} \Lambda & d_{12} \Lambda & d_{13} \Lambda \\
d_{12} \Lambda & d_{22} \Lambda & d_{23} \Lambda \\
d_{13} \Lambda & d_{23} \Lambda & d_{33} \Lambda
\end{array}\right]
$$

where the $d_{i j}$ are the components of the $\mathbf{D}_{b}$ matrix, equation (10), and

$$
\mathbf{\Lambda}=\boldsymbol{\phi}^{-1}=\frac{1}{A\left(\alpha \gamma-\psi^{2}\right)}\left[\begin{array}{ccc}
\alpha \gamma-\psi^{2} & 0 & 0 \\
0 & A \gamma & -A \psi \\
0 & -A \psi & A \alpha
\end{array}\right]
$$

The procedure used here to define $\mathbf{H}^{-1}$ is found to be valid and very effective for a general $\mathbf{D}_{b}$ matrix. The technique is similar to that used in Reference 9. The only difference is that in Reference 9, area co-ordinates are used instead of the $x$ and $y$ co-ordinates, equations (40)-(42). The result is that in Reference 9 the $\Lambda$ matrix is simpler in form than equation (50); however, the $\mathbf{G}$ matrix, as introduced in the discussion below, is more difficult to formulate. This is due to the different nature of the generalized parameters $\boldsymbol{\beta}$ in both approaches.

The second important step in the formulation of the stiffness matrix is the evaluation of the integral on $\partial A=12+23+31$. On side $i j$ ( $i j=12$, or 23 , or 31 ) we have

$$
U_{2}=\int_{i j}\left\{w Q_{n}-w, M_{n}-w, M_{n s}\right\} \mathrm{d} s
$$

The components $Q_{n}$ and $M_{n}$ and $M_{n s}$ are given in equation (37) (Figure 3):

$$
\mathrm{c}=\cos \gamma_{i j}=-y_{i i} / l_{i j} ; \quad \mathrm{s}=\sin \gamma_{i j}=x_{i j} / l_{i j}
$$

Using equations (37) and (40) it follows that

$$
\left[\begin{array}{c}
Q_{n} \\
-M_{n} \\
-M_{n s}
\end{array}\right]_{i j}=\mathbf{R}_{i j} \mathbf{\beta}
$$

where

$$
\mathbf{R}_{i j}=\left[\begin{array}{ccccccccc}
0 & c & 0 & 0 & 0 & s & 0 & s & c \\
-c c & -c c x & -c c y & -s s & -s s x & -s s y & -2 c s & -2 c s x & -2 c s y \\
c s & c s x & c s y & -c s & -c s x & -c s y & -(c c-s s) & -(c c-s s) x & -(c c-s s) y
\end{array}\right]
$$

with $x=x_{i}-\xi x_{i j}$ and $y=y_{i}-\xi y_{i j}$ and $\xi=s / l_{i j}$ (Figure 3). Thus, $\mathbf{R}_{i j}$ is a linear expression in $\xi$. The transverse displacement $w$ along the side $i j$ is expressed as a cubic:

$$
w=H_{01}^{1}(\xi) w_{i}+H_{02}^{1}(\xi) w_{j}+H_{11}^{1}(\xi) w, s_{i}+H_{12}^{1}(\xi) w_{, s_{i}}
$$


where

$$
\begin{array}{ll}
H_{01}^{1}=1-3 \xi^{2}+2 \xi^{3} & H_{02}^{1}=3 \xi^{2}-2 \xi^{3} \\
H_{11}^{1}=l_{i j}\left(\xi-2 \xi^{2}+\xi^{3}\right) & H_{12}^{1}=l_{i j}\left(-\xi^{2}+\xi^{3}\right)
\end{array}
$$

The normal slope $w_{, n}$ is assumed to vary linearly:

$$
w_{, n}=(1-\xi) w_{n_{i}}+\xi w_{n_{i}}
$$

Using equations (25), (55) and (57) we have

$$
\left[\begin{array}{c}
w \\
w, n \\
w, s
\end{array}\right]=\mathbf{L}_{i i} \mathbf{U}_{i j}
$$

where

$$
\mathbf{U}_{i j}=\left[\begin{array}{llllll}
w_{i} & \theta_{x_{i}} & \theta_{y_{i}} & w_{i} & \theta_{x_{i}} & \theta_{y_{i}}
\end{array}\right]
$$

and

$$
\mathbf{L}_{i j}=\left[\begin{array}{cccccc}
H_{01}^{1} & c H_{11}^{1} & s H_{11}^{1} & H_{02}^{1} & c H_{12}^{1} & s H_{12}^{1} \\
0 & s(1-\xi) & -c(1-\xi) & 0 & s \xi & -c \xi \\
\frac{H_{01, \xi}^{1}}{l_{i j}} & \frac{c H_{11, \xi}^{1}}{l_{i j}} & \frac{s H_{11, \xi}^{1}}{l_{i j}} & \frac{H_{02, \xi}^{1}}{l_{i j}} & \frac{c H_{12, \xi}^{1}}{l_{i j}} & \frac{s H_{12, \xi}^{1}}{l_{i j}}
\end{array}\right]
$$

Using equations (53) and (58), equation (51) becomes

$$
U_{2}=\boldsymbol{\beta}^{\mathrm{T}} \mathbf{G}_{i j} \mathbf{U}_{i j}
$$

where

$$
\mathbf{G}_{i j}=\int_{0}^{l_{i d}} \mathbf{R}_{i j}^{\mathrm{T}} \mathbf{L}_{i j} \mathrm{~d} s=l_{i j} \int_{0}^{1} \mathbf{R}_{i j}^{\mathrm{T}}(\xi) \mathbf{L}_{i j}(\xi) \mathrm{d} \xi
$$

$\mathbf{G}_{i j}$ is a $9 \times 6$ matrix and the integration is performed in closed form. The compact and explicit expression of $\mathbf{G}_{i j}$ is given in Appendix B.

A $9 \times 9$ matrix $\mathbf{G}$ can be defined such that

$$
U_{3}=\int_{\partial A}\left\{w Q_{n}-w, M_{n}-w, M_{n s}\right\} \mathrm{d} s=\beta^{\mathrm{T}} \mathbf{G U}
$$

with

$$
\mathbf{G U}=\mathbf{G}_{12} \mathbf{U}_{12}+\mathbf{G}_{23} \mathbf{U}_{23}+\mathbf{G}_{31} \mathbf{U}_{31}
$$

The matrix expression for equation (34) is therefore

$$
U_{\mathrm{HS}}=-\frac{1}{2} \boldsymbol{\beta}^{\mathbf{T}} \mathbf{H} \boldsymbol{\beta}+\boldsymbol{\beta}^{\mathrm{T}} \mathbf{G U}
$$

The stresses (associated with $\beta$ ) vary independently within the elements and the displacements (associated with $\mathbf{U}$ ) are common to adjacent elements. Therefore we have

$$
\boldsymbol{\beta}=\mathbf{H}^{-1} \mathbf{G U}
$$


and by analogy with the conventional displacement method the stiffness matrix of the HSM element becomes

$$
\mathbf{K}_{\mathbf{H S M}}=\mathbf{G}^{\mathbf{T}} \mathbf{H}^{-1} \mathbf{G}
$$

Once the nodal displacements are known, the bending moments are evaluated using equations (40) and (66):

$$
\mathbf{M}=\mathbf{P}(x, y) \mathbf{H}^{-1} \mathbf{G U}
$$

Note that different bending moments are obtained along the interelement boundaries since the $\beta$ 's are different for different elements.

\subsection{The selective reduced integration element (SRI)}

The formulation of the SRI element is based on the theory of plates with transverse shear deformations included (see Section 3.1.1) and follows the approach given in References 42 and 54. The main difficulty in the development of selectively integrated elements is to obtain an accurate and reliable element. The issue of reliability is most important in actual practical analysis, ${ }^{58}$ and we would only want to use an element that does not contain spurious zero energy modes.

The basic displacement assumptions for the SRI element are the deformations $w, \beta_{x}$ and $\beta_{y}$ which vary linearly over the element:

$$
w=\sum_{i=1}^{3} L_{i} w_{i} \quad \beta_{x}=\sum_{i=1}^{3} L_{i} \beta_{x_{i}} \quad \beta_{y}=\sum_{i=1}^{3} L_{i} \beta_{y_{i}}
$$

where $L_{i}=1-\xi-\eta, L_{2}=\xi, L_{3}=\eta$ and $w_{i}, \beta_{x_{i}}$ and $\beta_{y_{i}}, i=1,2,3$, are the nine nodal values at the corner nodes (Figure 1).

Using equations (7), (14) and (18) the stiffness matrix of the SRI element is defined as

$$
\mathbf{K}_{\mathrm{SRI}}=\mathbf{K}_{b}+\mathbf{K}_{s}
$$

where $\mathbf{K}_{b}$ represents the stiffness matrix due to bending, and $K_{s}$ the stiffness matrix due to transverse shear,

$$
\mathbf{K}_{b}=2 A \int_{A} \mathbf{B}_{b}^{\mathrm{T}} \mathbf{D}_{b} \mathbf{B}_{b} \mathrm{~d} \xi \mathrm{d} \eta
$$

where $\mathbf{D}_{b}$ is given in equations (10) or (12a). Also,

$$
\mathbf{B}_{b}=\frac{1}{2 A}\left[\begin{array}{ccccccccc}
0 & 0 & y_{23} & 0 & 0 & y_{31} & 0 & 0 & y_{12} \\
0 & x_{23} & 0 & 0 & x_{31} & 0 & 0 & x_{12} & 0 \\
0 & -y_{23} & -x_{23} & 0 & -y_{31} & -x_{31} & 0 & -y_{12} & -x_{12}
\end{array}\right]
$$

and

$$
\mathbf{K}_{s}=2 A \int_{A} \mathbf{B}_{s}^{\mathrm{T}} \mathbf{D}_{s} \mathbf{B}_{s} \mathrm{~d} \xi \mathrm{d} \eta
$$

where $D_{s}$ is given in equations $(11)$ or $(12 b)$, and

$$
\mathbf{B}_{s}=\frac{1}{2 A}\left[\begin{array}{ccccccccc}
y_{23} & 0 & 2 A L_{1} & y_{31} & 0 & 2 A L_{2} & y_{12} & 0 & 2 A L_{3} \\
-x_{23} & -2 A L_{1} & 0 & -x_{31} & -2 A L_{2} & 0 & -x_{12} & -2 A L_{3} & 0
\end{array}\right]
$$


In equation (70) $\mathbf{K}_{b}$ is a matrix with constant terms (exact integration), and $\mathbf{K}_{s}$ is a matrix involving integrals of quadratic terms in $\xi, \eta$. If exact integration is used, convergence towards the thick plate solution is obtained but the rate of convergence is low. Considering the analysis of thin plates the concept of reduced integration can be employed ${ }^{42,54}$ by evaluating the shear stiffness $\mathbf{K}_{s}$ using only one numerical integration point (which is equivalent to assuming that $\boldsymbol{\gamma}$ is constant over the element). This element has been tested and the numerical results obtained in the analysis of some problems that are given in Section 4.3. These results show that the element is not effective in the analysis of thin plates. In addition, the element also contains spurious zero energy modes, which would have to be eliminated before the element could be recommended for general practical analysis.

As established in recent studies, ${ }^{45.56}$ in certain cases the stiffness matrix associated with a selective integration formulation is equivalent to the stiffness matrix obtained using a mixed formulation of the Hellinger-Reissner type, where the dof associated with the stresses are considered as internal (element) parameters. If this equivalence can be shown, it provides further insight into the formulation of the element. For the present triangular element we show this equivalence in Appendix C.

\section{IMPLEMENTATION AND NUMERICAL RESULTS}

The DKT, HSM and SRI plate bending elements have been implemented in the computer program ADINA. ${ }^{49}$ Several sample problems have been solved using these elements and the results have been compared with those obtained using other elements.

\subsection{Remarks on the implementation of the elements}

The calculation of the DKT and SRI elements is similar to that of the two-dimensional isoparametric plane-stress element (evaluation of $\mathbf{B}$ matrix and integration of $\mathbf{B}^{\mathrm{T}} \mathbf{D}_{b} \mathbf{B}$, Reference 11). In the case of the HSM element, the matrices $\mathbf{H}^{-1}$ and $\mathbf{G}$ are first evaluated. The stiffness matrix is then obtained using equation (67).

Considering the loading on the elements, a uniform loading is represented by lumped concentrated loads acting at the nodes, i.e. the element load vector corresponding to a uniform pressure load $q$ per unit middle surface is

$$
\mathbf{f}^{\mathrm{T}}=A / 3\left[\begin{array}{lllllllll}
q & 0 & 0 & q & 0 & 0 & q & 0 & 0
\end{array}\right]
$$

The bending moments are evaluated using equations (32) and (68) for the DKT and HSM elements, respectively. In ADINA, the moments can be evaluated at a maximum of seven points: the centroid, the three corner nodes and the three mid-side nodes of each element.

In dynamic analysis, a diagonal lumped mass matrix is employed. Neglecting the inertia effects at the rotational dof, the vector of the diagonal elements of the mass matrix is given in equation (75) with $\rho$ instead of $q$, where $\rho$ is the mass density per unit area. The techniques and strategies used to evaluate the natural frequencies are those of the ADINA program. ${ }^{49}$

A study of the formulations given in Section 3 shows that comparable storage and operations are needed to form the stiffness matrix of the HSM element compared to that of the DKT element. This is also true for the evaluation of the stresses in the elements.

\subsection{Analysis of sample problems using the DKT and HSM elements}

Several sample problems were analysed using both the DKT and HSM elements. The element performances were evaluated and compared with those of other 9 dof elements. 
The analysis results of different static and free vibration problems are described in the following sections. Some of the results presented have been reported already by other authors, e.g. References 19, 33, 36 and 59 for the DKT element, and References 26, 27 and 28 for the HSM element.

4.2.1. Square plate under concentrated and uniform loads with clamped and simply-supported edges. In this analysis, a square plate of sides $2 a$ with either simply-supported or clamped edges is considered. Owing to symmetry in the problem, only one-quarter of the plate is modelled. The plate and the two different mesh orientations (A and B) used in the analysis are illustrated in Figure 5. Four different sizes of meshes are considered: 2, 8, 32 and 128 triangular elements are
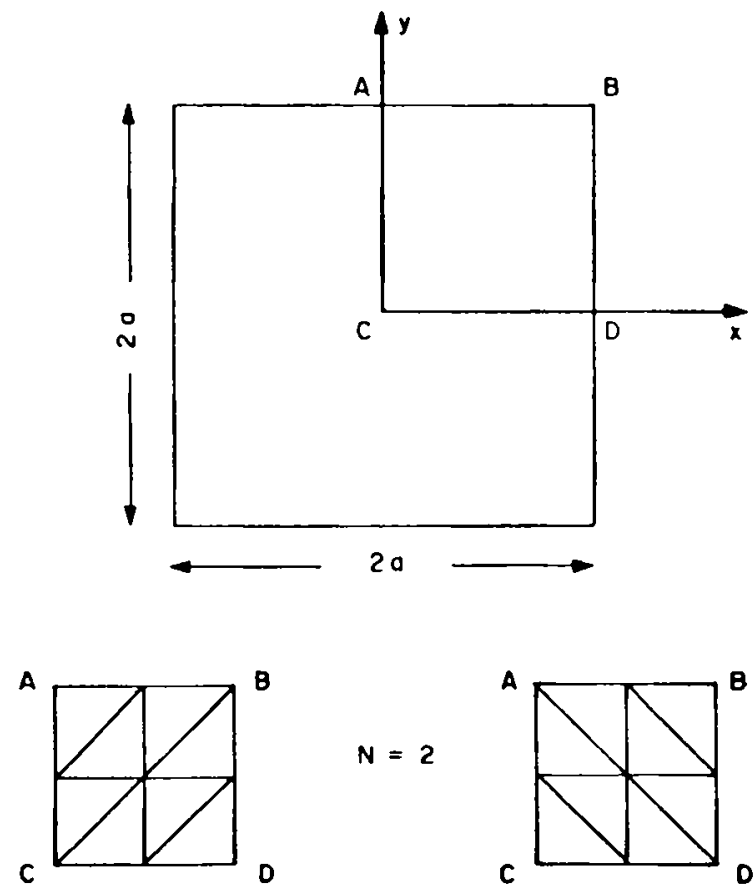

MESH ORIENTATION A

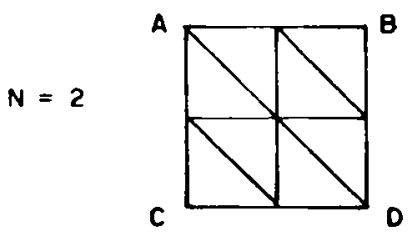

MESH ORIENTATION $B$

\begin{tabular}{|l|c|c|c|c|}
\hline ELEMENT & REFERENCE & SYMBOL & MESH & UNIFORM LOAD \\
\hline DKT & $33-36$ & 0 & A, B & lumped \\
HSM & $25-27$ & 0 & $A, B$ & lumped \\
BCIZ1 & 17 & $\bullet$ & $A$ & lumped \\
BCIZ2 & 17.30 & - & $A$ & lumped \\
HCT & 16 & $\Delta$ & $B$ & lumped \\
A-9 & 21,31 & $\nabla$ & $A$ & consislent \\
STRUDL & 22 & $*$ & $A$ & lumped \\
\hline
\end{tabular}

Figure 5. Square plate and mesh orientations 
used to form the meshes corresponding to $N=1,2,4$ and 8 , respectively. For the case of the concentrated load $(P)$, the different sizes of meshes in both $A$ and $B$ orientations are employed while only mesh orientation $A$ is considered for the case of the uniform pressure loading $(q)$. In all cases, the simply-supported as well as the clamped boundary conditions are considered.

As shown in Figure 5, both the DKT and HSM elements are evaluated with other established elements, namely the BCIZ1, BCIZ2, HCT, A-9 and STRUDL elements. The central deflections and moments of the DKT and HSM elements for all the above cases are given in Reference 53. The comparisons of the predicted results with those of other elements are given in Figures 6-15. Some remarks on the results are given in the following:

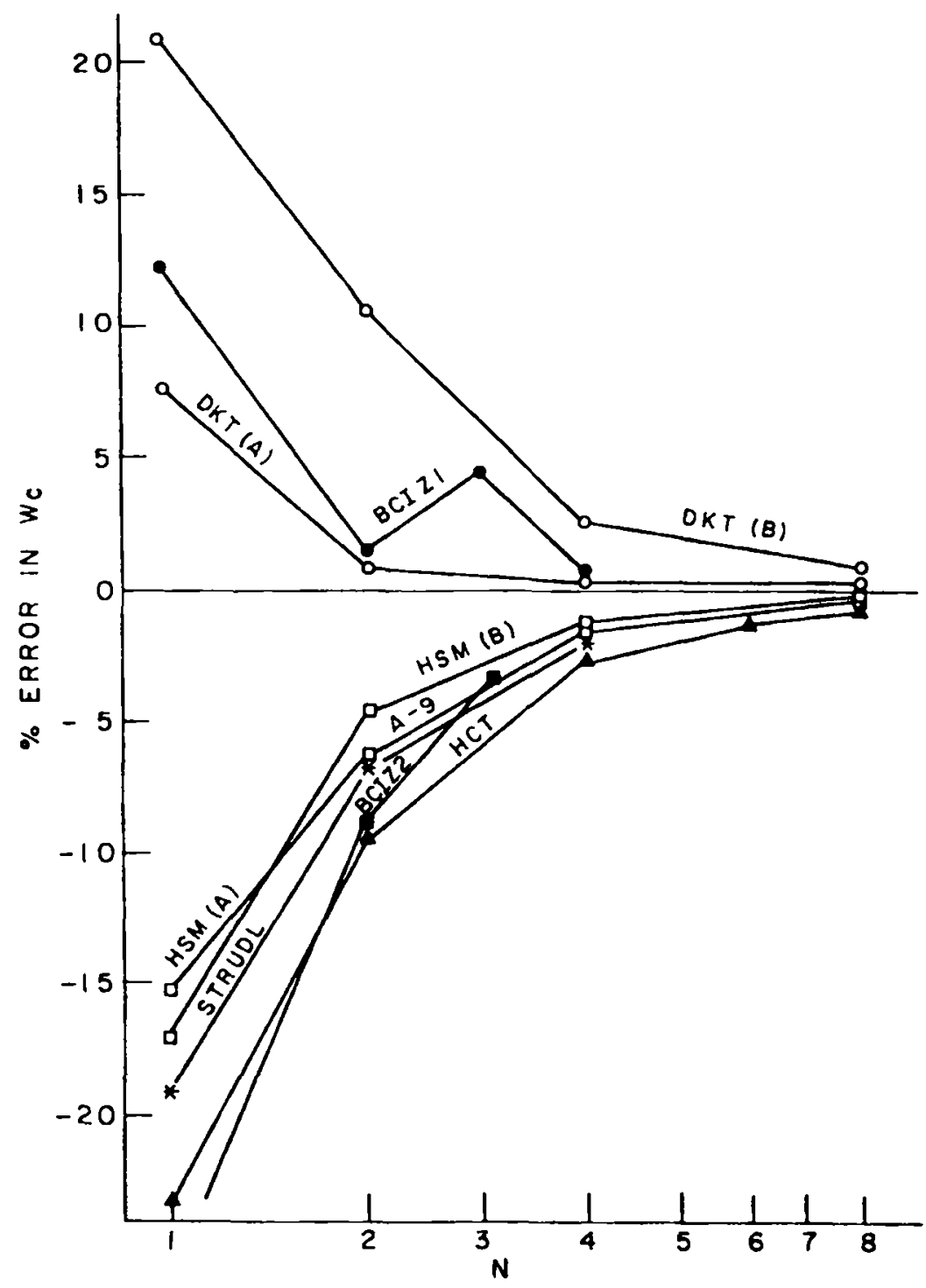

Figure 6. Simply-supported plate with concentrated load : error in deflection at centre 
(1) Considering first the case of the concentrated load application, Figures 6 and 7 show that the DKT and HSM elements are very efficient. Note that mesh $B$ is not effective in modelling the clamped plate problem, since all dofs of the corner element vanish. The influence of mesh orientation on the displacements is more severe for the DKT element than for the HSM element. Convergence from above is obtained for the DKT element, whereas monotonic convergence from below is observed for the HSM element.

(2) In the case of uniform loading, monotonic convergence was achieved with the DKT element with both boundary conditions. However, convergence is less rapid in the case of the clamped plate. The solution accuracy could probably be increased by employing a consistent load representation with a cubic polynomial for $w$. Monotonic convergence is not achieved in the analysis of the clamped plate using the HSM element.

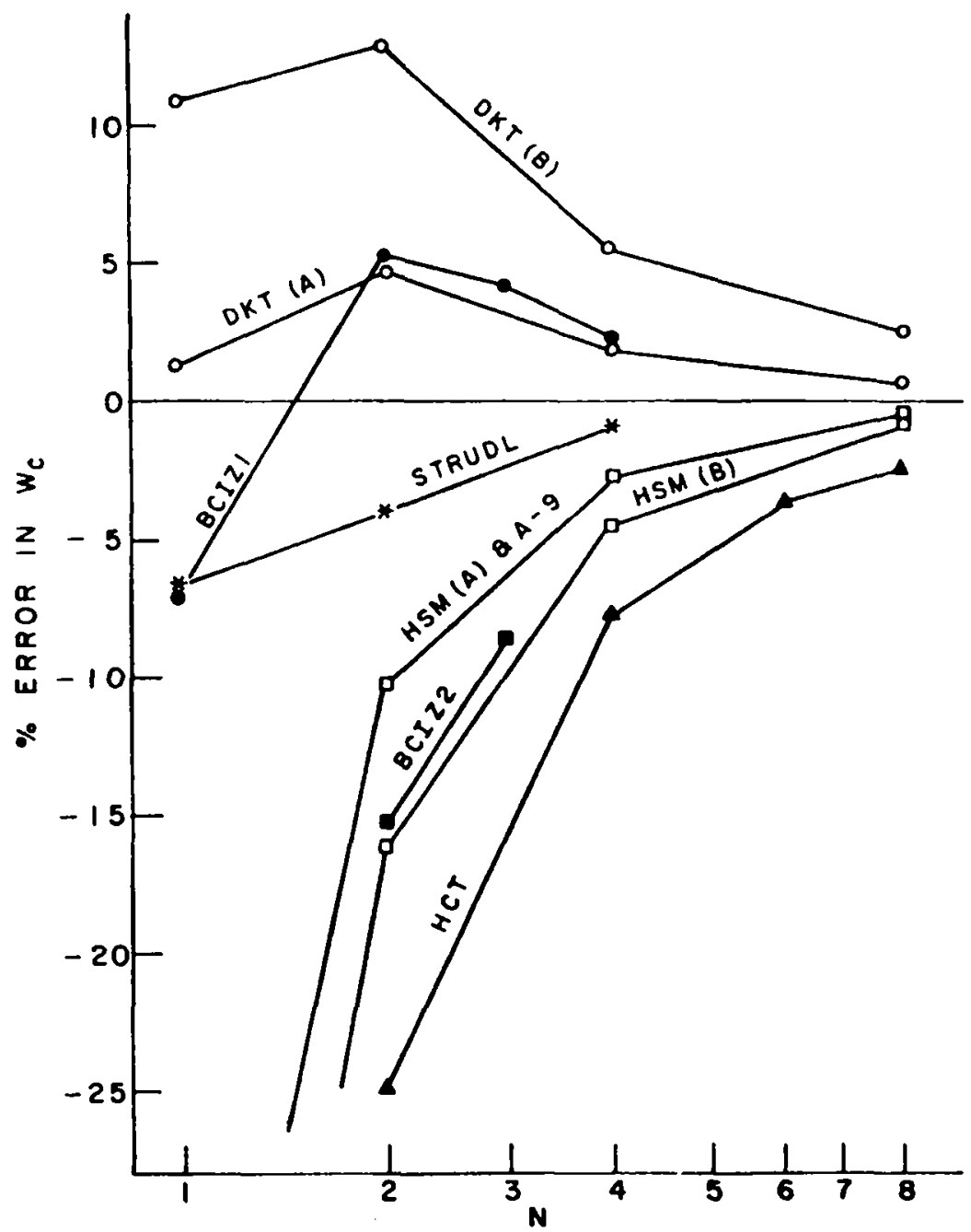

Figure 7. Clamped plate with concentrated load: error in deflection at centre 


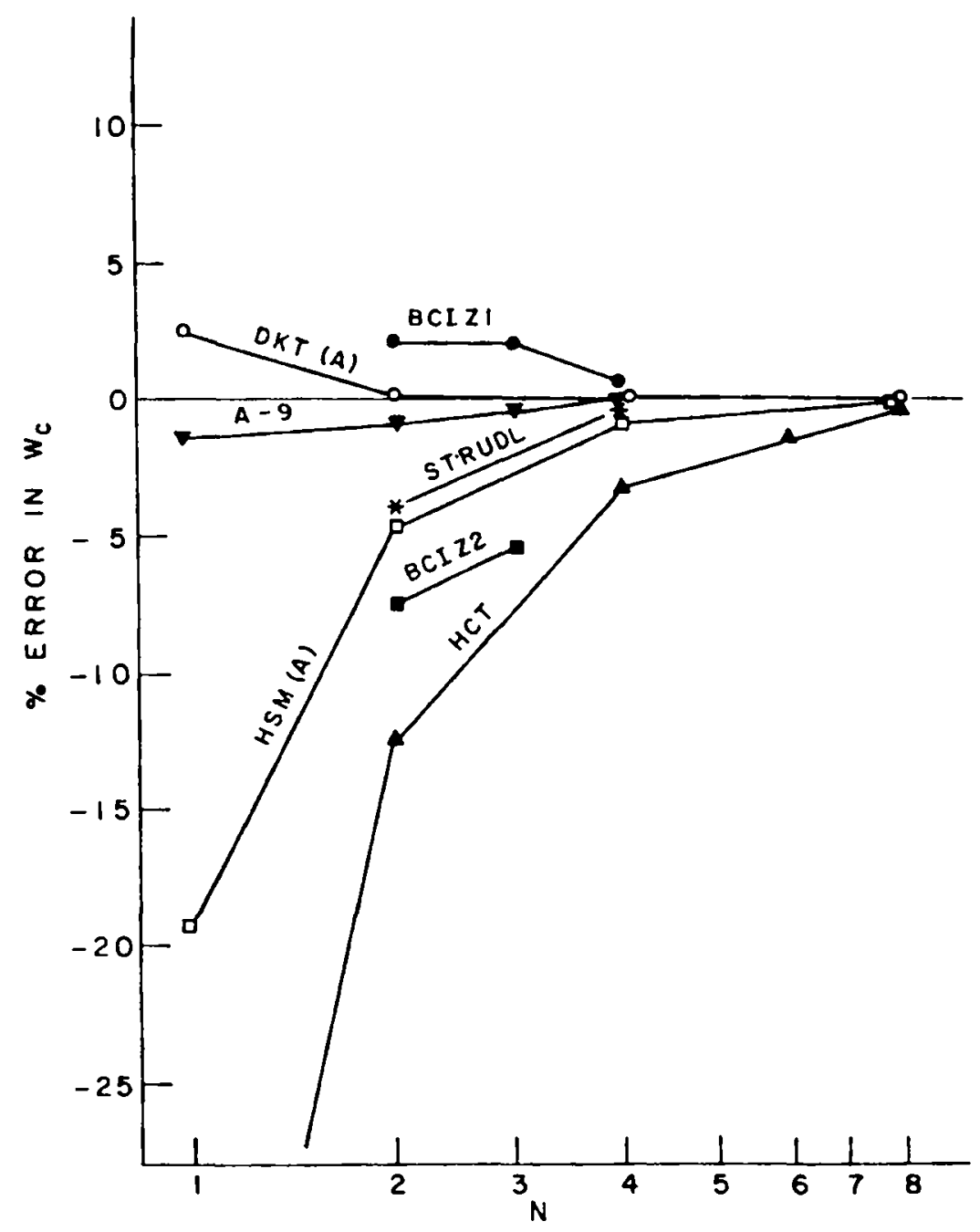

Figure 8. Simply-supported plate with uniform loading : error in deflection at centre

(3) In general, the stresses obtained with the HSM element meshes are slightly better than those with the DKT elements for the same problem (see Figures 10-15, and Tables 4-7 of Reference 53). Similarly, the boundary conditions in terms of stresses such as $M_{n}=0$ along a simplysupported side are slightly better satisfied with the HSM elements. However, the difference between two distinct values of moments at a node connecting two elements is not smaller with the HSM elements.

4.2.2. Twisting of a square plate. This study is intended to evaluate the twisting behaviour of the DKT and HSM elements. Particular attention is given to the DKT element as the twisting curvatures due to $\beta_{x, y}$ and $\beta_{y, x}$ are not equal in general, equation (3). This problem can, therefore, be used to evaluate the ability of the DKT element to represent stresses due to a constant twist (a patch test problem). The same problem has been considered in References 16 and 21 using other finite elements. 


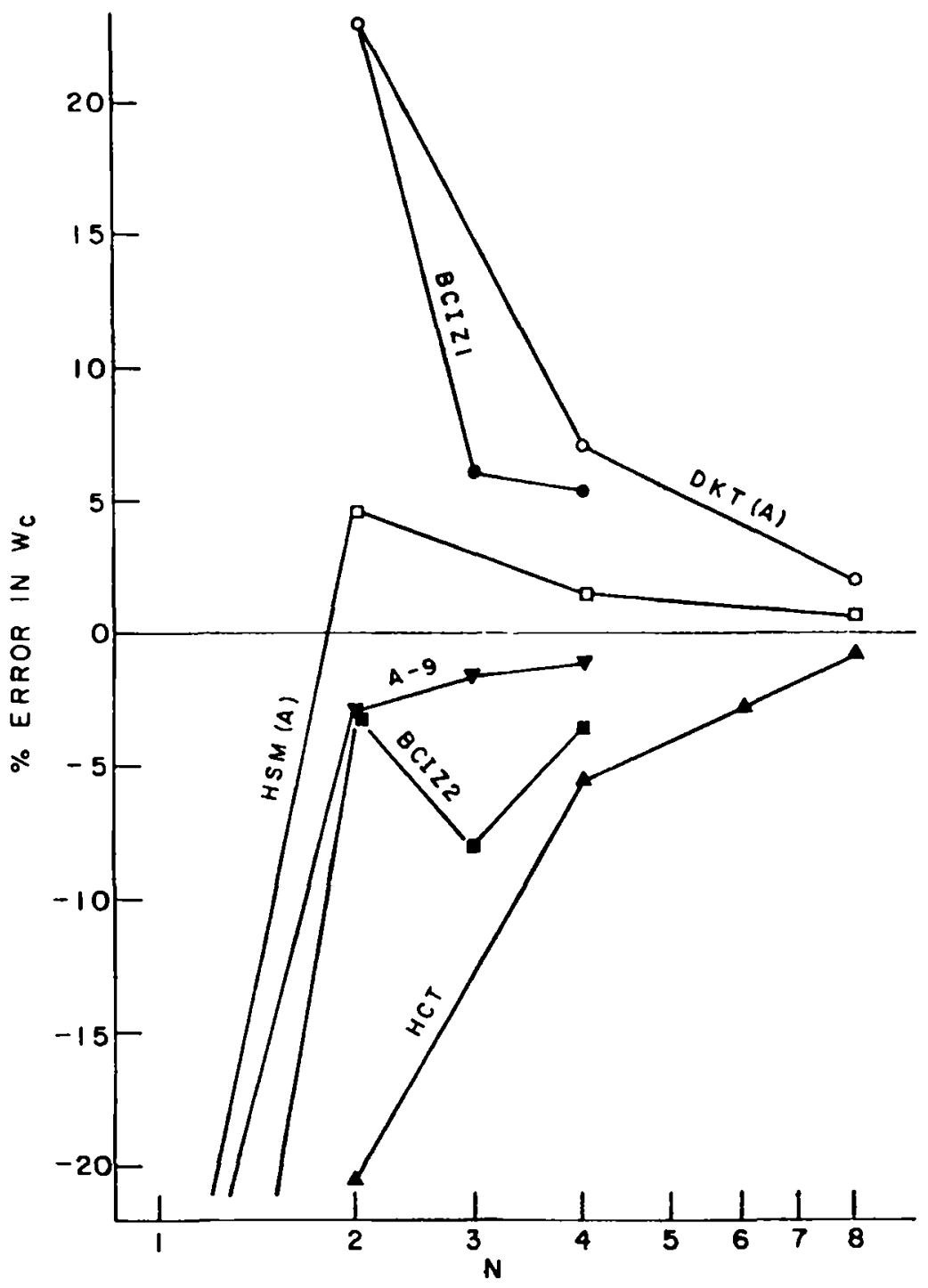

Figure 9. Clamped plate with uniform loading : error in deflection at centre

The square plate is simply supported (i.e. $w=0$ ) at the corners $A, B$ and $D$ as shown in Figure 16. A vertical load is applied at corner $C$. The exact thin plate solution with the data given in Figure 16 is $M_{x}=M_{y}=0$ and $M_{x y}=2.5 \mathrm{lb}$-in/in everywhere in the plate with $w_{c}=0.2496$ in and $w_{0}=0.0624$ in.

Four different meshes are considered: meshes (a) and (b) are regular with 2 and 4 elements, respectively; meshes (c) and (d) are irregular with 4 and 8 elements, respectively.

The results obtained using both the DKT and HSM elements are excellent. All four meshes with either element give the exact solution for stresses and displacements (stresses are evaluated at the centroid as well as at the corner nodes). These results are compared in Figure 16 with those given in Reference 16. The ACM (a 12 dof incompatible rectangular element) and the HCT 


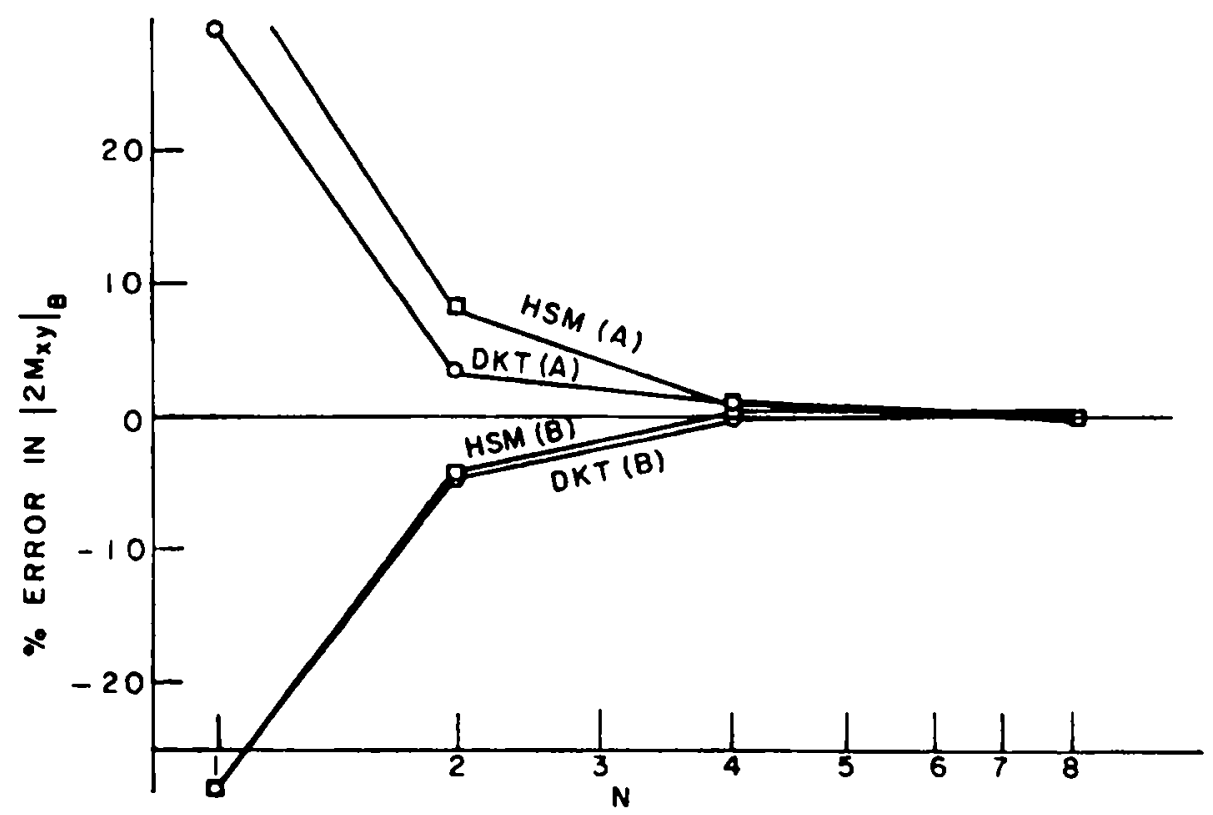

Figure 10. Simply-supported plate with concentrated load : error in corner reaction

results were obtained with a regular fine mesh of $8 \times 8$ elements. Considering the results given in Reference 21, the A-9 and the non-conforming BCIZ1 elements do reproduce the exact stresses with mesh (a), and the BCIZ1 element cannot predict the exact solution even with very fine meshes if the mesh subdivision is irregular. (A 30 per cent error in the stresses exists at the centroid for mesh (d).)

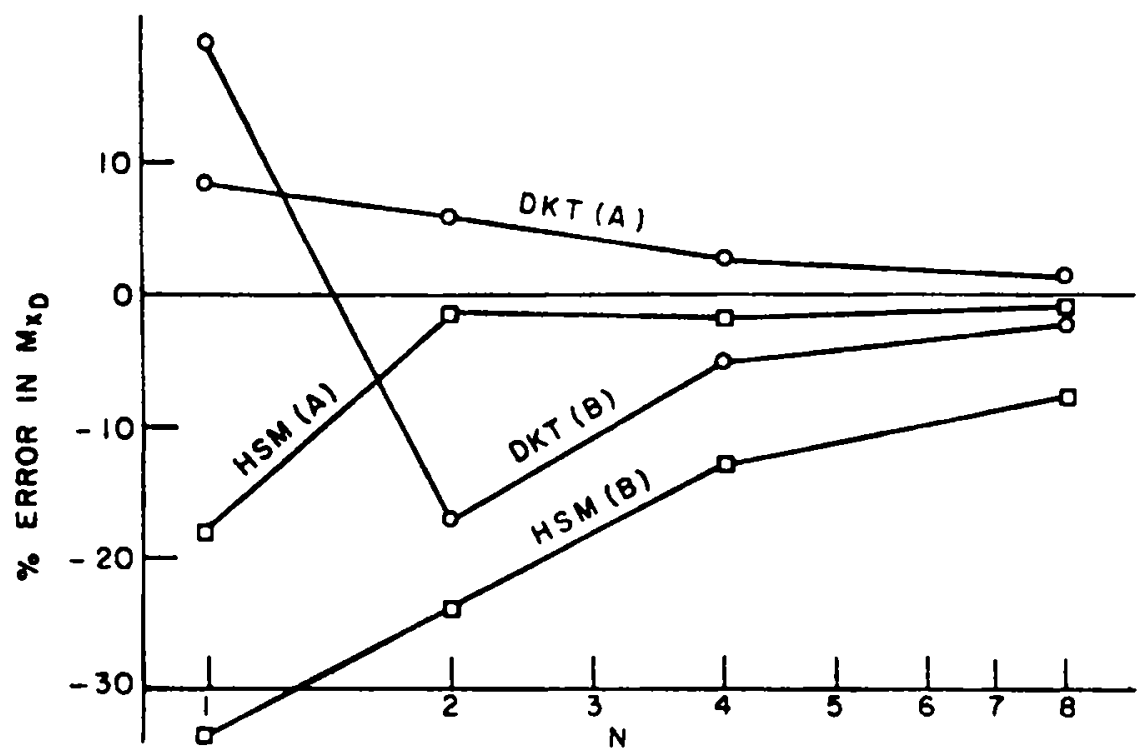

Figure 11. Clamped plate with concentrated load: error in bending moment at centre of side 


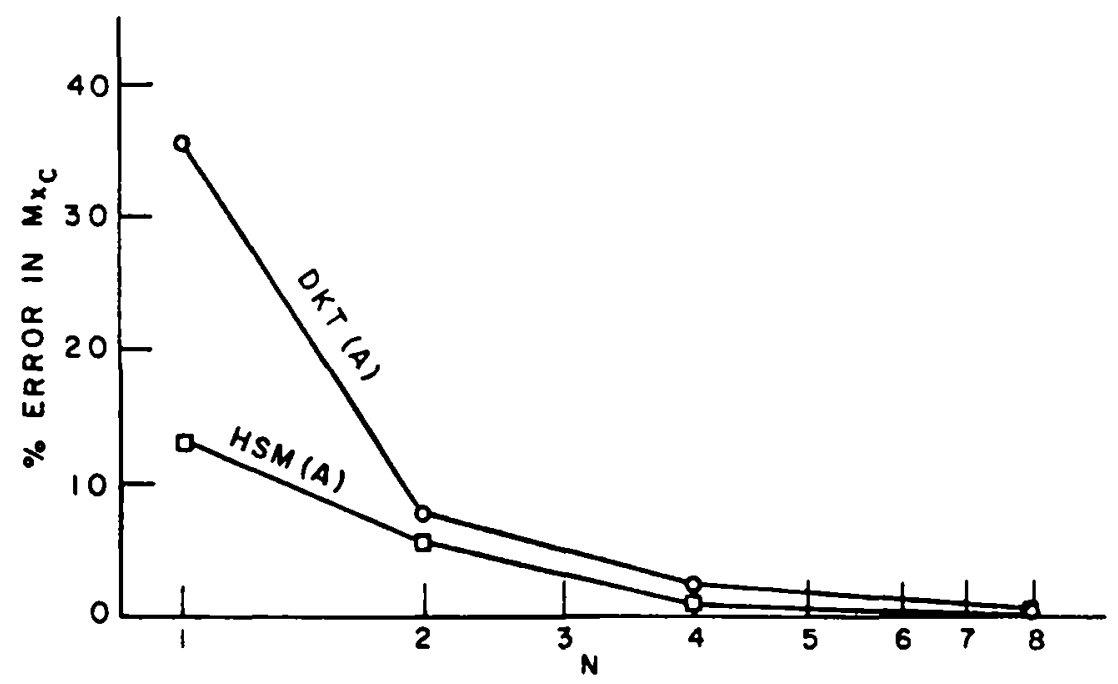

Figure 12. Simply-supported plate with uniform loading: error in bending moment at centre

4.2.3. Static analysis of a rhombic cantilever. This problem deals with the analysis of a rhombic cantilevered plate subjected to a uniform load. The geometry and material properties are given in Figure 17. Experimental results of this problem are available for comparison. ${ }^{16}$

A $4 \times 4$ mesh is used and the results obtained with both the DKT and HSM elements are given in Figure 17. It is observed that even with this coarse mesh, the DKT element gives results in good agreement with the experimental values.

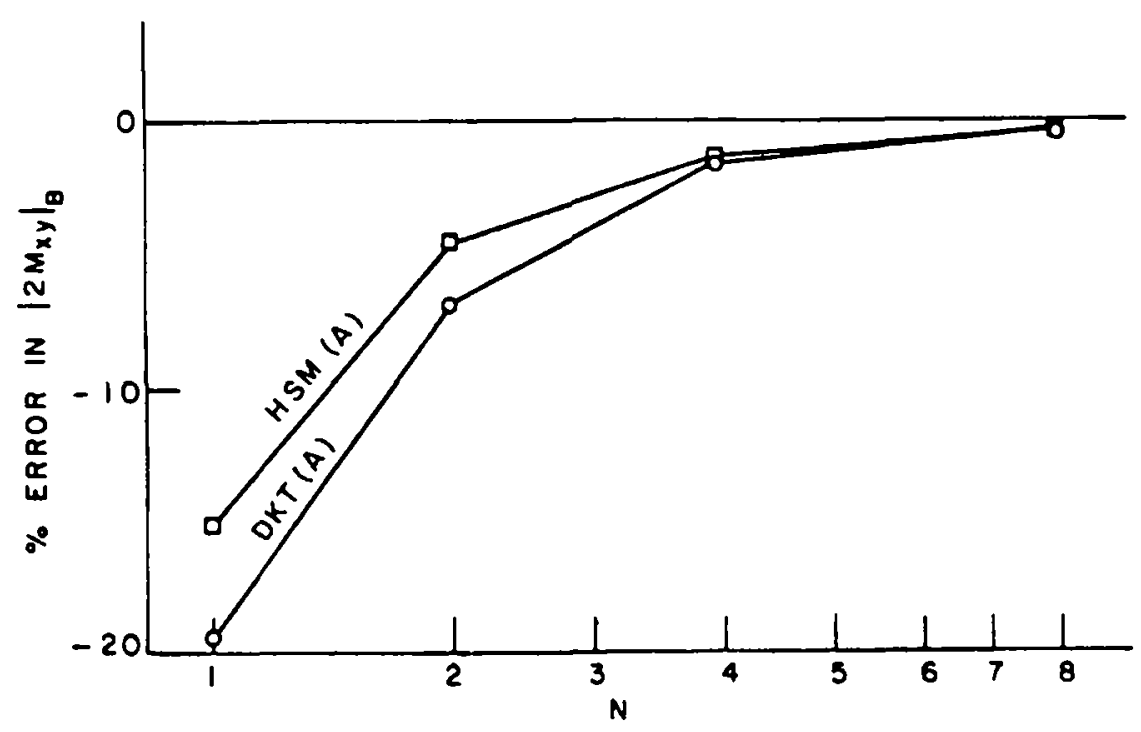

Figure 13. Simply-supported plate with uniform loading: error in corner reaction 


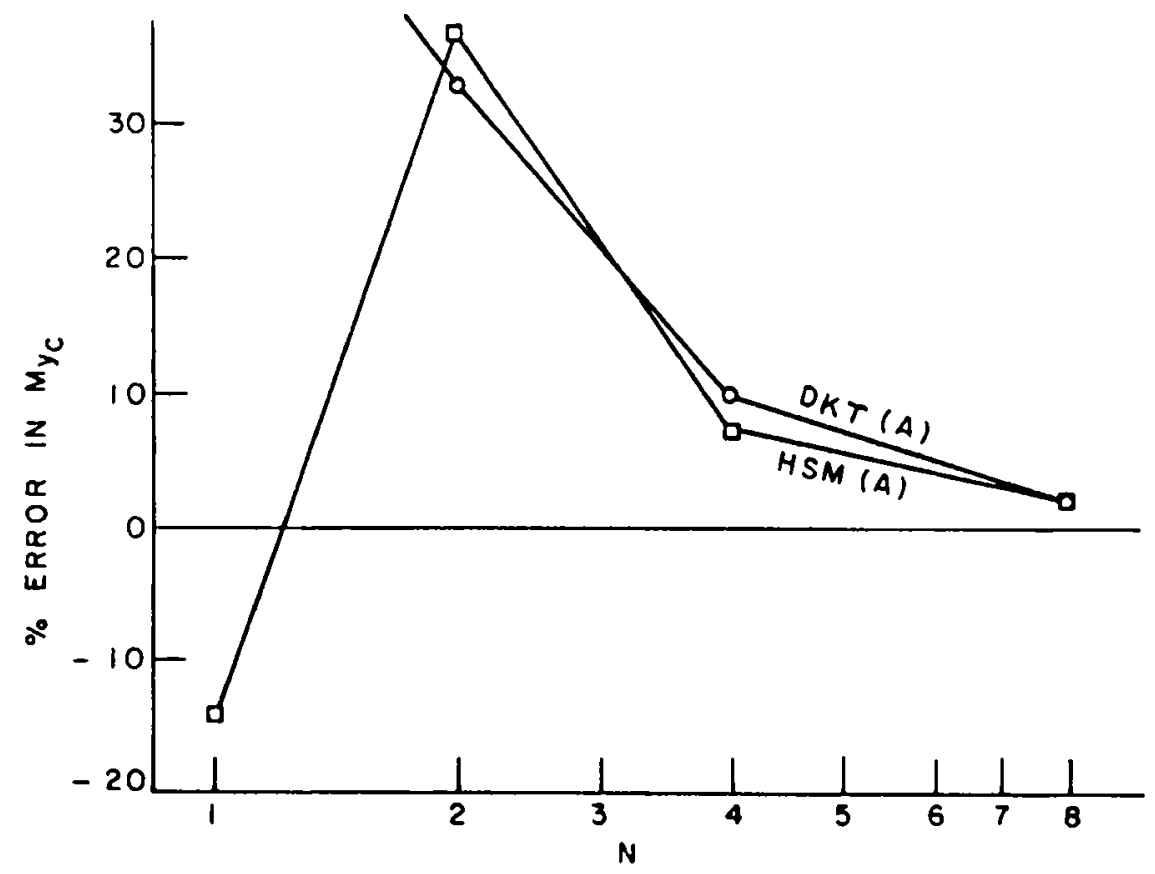

Figure 14. Clamped plate with uniform loading: error in bending moment at centre

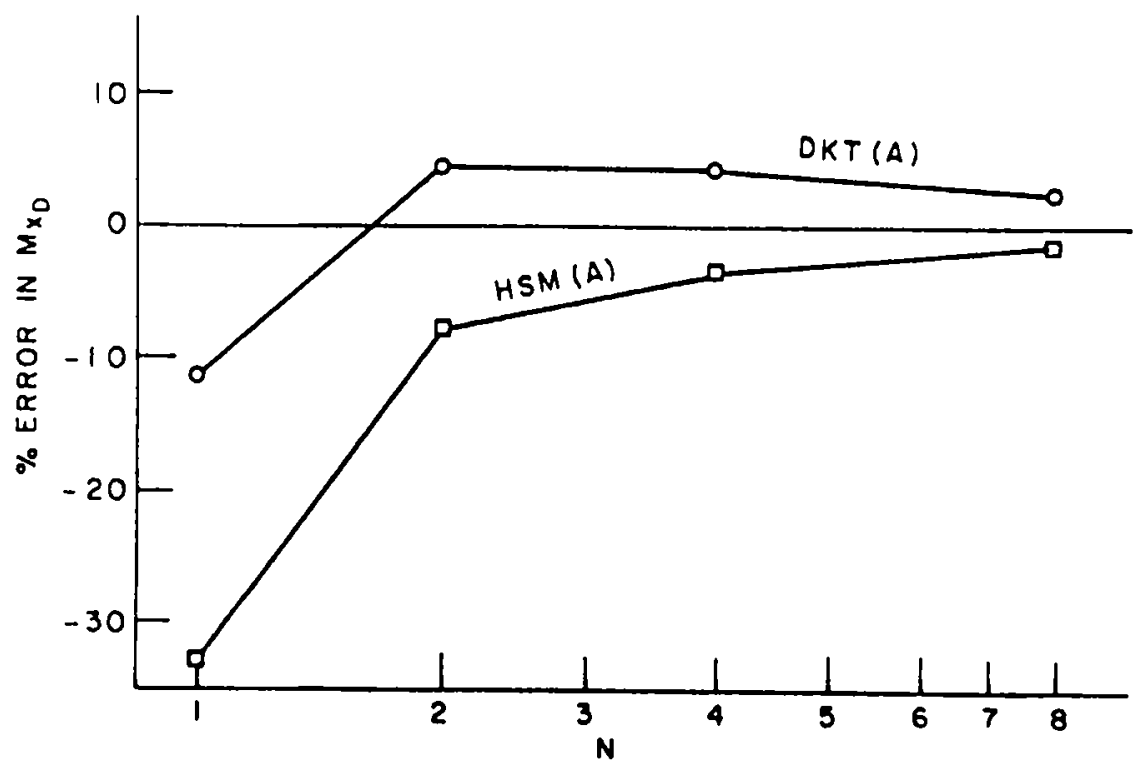

Figure 15. Clamped plate with uniform loading: error in bending moment at centre of side 


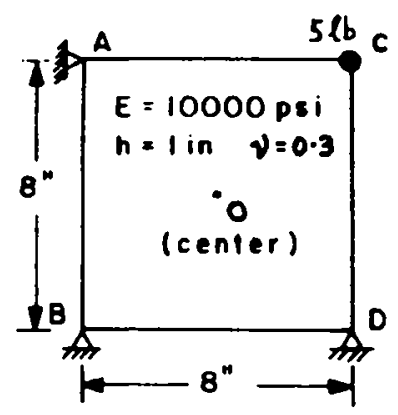

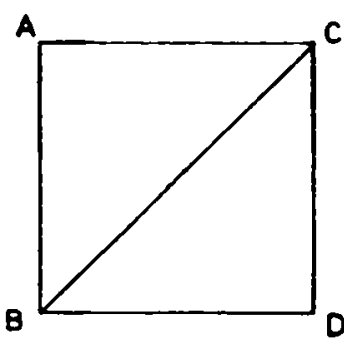

(a)

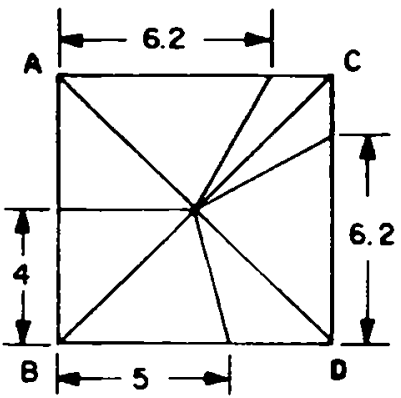

(d)

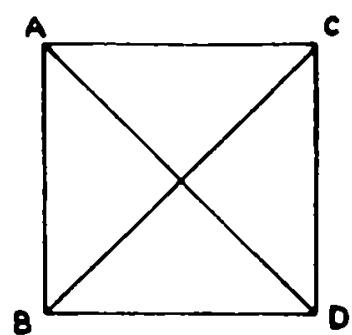

(b)

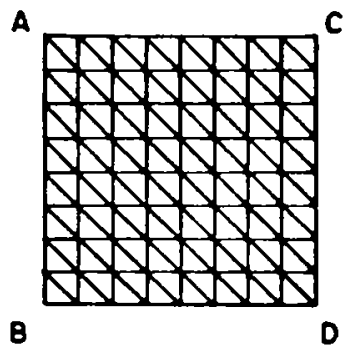

(e)

\begin{tabular}{|c|c|c|c|c|c|}
\hline \multirow{2}{*}{\multicolumn{2}{|c|}{ ELEMENT TYPE }} & \multicolumn{2}{|c|}{ DEFLECTION AT } & \multicolumn{2}{|c|}{ MOMENTS IN THE PLATE } \\
\hline & & POINT 0 & POINT C & $M_{x}$ and $M_{y}$ & $M_{x y}(i b-i n / i n)$ \\
\hline \multirow{4}{*}{ DKT } & MESH (a) & - & 0.24960 & $0^{*}$ & 2.50000 \\
\hline & MESH (b) & 0.06240 & 0.24960 & 0 & 2.50000 \\
\hline & MESH (c) & - & 0.24960 & 0 & 2.50000 \\
\hline & MESH (d) & 0.06240 & 0.24960 & 0 & 2.50000 \\
\hline \multirow{4}{*}{ HSM } & MESH (a) & - & 0.24960 & 0 & 2.50000 \\
\hline & MESH (b) & 0.06240 & 0.24960 & 0 & 2.50000 \\
\hline & MESH $(c)$ & - & 0.24960 & 0 & 2.50000 \\
\hline & MESH (d) & 0.06240 & 0.24960 & 0 & 2.50000 \\
\hline \multicolumn{2}{|c|}{ ACM $[16]$ MESH (e) } & 0.06244 & 0.24972 & - & - \\
\hline \multicolumn{2}{|c|}{ HCT [16] MESH (e) } & 0.06254 & 0.25002 & - & - \\
\hline \multicolumn{2}{|c|}{ EXACT VALUE } & 0.06240 & 0.24960 & 0 & 2.50000 \\
\hline
\end{tabular}

* To at least eight significant figures

Figure 16. Twisting of a square plate with comparison of numerical results 


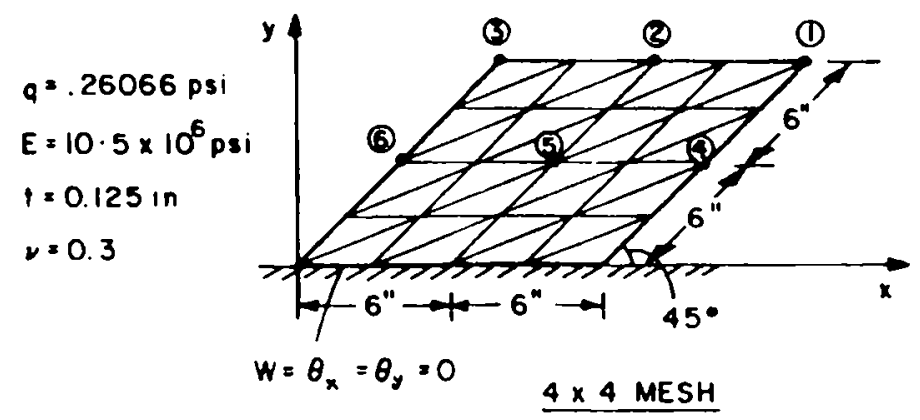

\begin{tabular}{|c|c|c|c|c|c|c|c|c|}
\hline \multirow{2}{*}{ ELENENT } & \multirow{2}{*}{ MESH } & \multirow{2}{*}{$\begin{array}{l}\text { TOTAL } \\
\text { NO. Or } \\
\text { DGF }\end{array}$} & \multicolumn{6}{|c|}{ DEFLECTION AT LOCATION } \\
\hline & & & 1 & 2 & 3 & 4 & 5 & 6 \\
\hline DKT & $4 \times 4$ & 75 & 0.304 & 0.198 & $0.1 ! 3$ & 0.121 & 0.055 & 0.022 \\
\hline & & & $(2.4)^{\star}$ & $(-2.9)$ & $(-6.6)$ & $(-6.2)$ & $(-0.7)$ & $(2.3)$ \\
\hline HSM & $4 \times 4$ & 75 & 0.264 & 0.173 & 0.100 & 0.095 & 0.043 & 0.021 \\
\hline & & & $(-11.3)$ & $(-15.2)$ & $(-17.8)$ & $(-25.4)$ & $(-22.5)$ & $\{-5.5\}$ \\
\hline ACM & $8 \times 6$ & 189 & 0.296 & 0.193 & 0.114 & 0.114 & 0.052 & 0.020 \\
\hline [16] & & & $(-0.3)$ & $(-2.0)$ & $(-0.2)$ & $(-11.6)$ & $(-7.1)$ & $(-10.5)$ \\
\hline HCT & $8 \times 6$ & 189 & 0.281 & 0.188 & 0.111 & 0.111 & $0.04 \subseteq$ & 0.018 \\
\hline$[16]$ & & & $(-5,3)$ & $(-7,2)$ & $(-8.4)$ & $(-13.8)$ & $(-12,3)$ & $(-17.3)$ \\
\hline \multicolumn{3}{|c|}{$\begin{array}{l}\text { EXPEPIMEITAL VALLEE } \\
\text { [16] }\end{array}$} & 0.237 & 0.204 & 0.121 & 0.129 & 0.055 & $0.5 \hat{z}=$ \\
\hline
\end{tabular}

- Percentage ceviation from exforimental value

Figure 17. Rhombic cantilever plate with comparison between numerical and experimental results

4.2.4. Free vibration analysis of a square plate with clamped and simply-supported edges. The natural frequencies and mode shapes of simply-supported and clamped square plates are evaluated using the DKT and HSM elements and a lumped diagonal mass matrix. Two uniform mesh orientations (mesh $A$ and $B$, Figure 5) are used with $N=1,2,4,8$. Only doubly-symmetric modes were calculated by considering one-quarter of the plate (ABCD in Figure 5).

The first six eigenvalues (and associated modes) are given in Tables I and II. The modes are described by the number of half-waves $m$ and $n$ parallel to the $x$ and $y$ axes, respectively: $(1,1)$ corresponds to the fundamental mode with no nodal line within the plate. The non-dimensional eigenvalue is $\lambda=\rho \omega^{2}\left[(2 a)^{4}\right] / D$, where $\rho$ is the mass density per unit mid-surface and $\omega$ the angular frequency. For the simply-supported plate the exact successive eigenvalues are given by 
Table I. Free vibration frequencies of simply-supported square plate (symmetric modes)

\begin{tabular}{|c|c|c|c|c|c|c|c|}
\hline Element & $\begin{array}{l}\text { Mesh } \\
\text { orien- } \\
\text { tation }\end{array}$ & Mode & 1 & 2 & 4 & 8 & $\begin{array}{c}\text { Analytical } \\
\lambda=\left(m^{2}+n^{2}\right)^{2} \pi^{4}\end{array}$ \\
\hline \multirow[t]{2}{*}{ DKT } & A & $\begin{array}{l}(1,1) \\
(1,3) \\
(3,1) \\
(3,3) \\
(1,5) \\
(5,1)\end{array}$ & $240 \cdot 20^{+}$ & $\begin{array}{r}344.54 \\
6,872 \cdot 42 \\
7,240 \cdot 92 \\
14,500 \cdot 13\end{array}$ & $\begin{array}{r}377 \cdot 29 \\
9,126 \cdot 28 \\
9,388 \cdot 30 \\
27,264 \cdot 37 \\
57,129 \cdot 63 \\
58,768 \cdot 28\end{array}$ & $\begin{array}{r}386 \cdot 46 \\
9,579 \cdot 76 \\
9,653 \cdot 93 \\
30,423 \cdot 29 \\
64,282 \cdot 79 \\
64,859 \cdot 60\end{array}$ & $\begin{aligned} \lambda_{1} & =389.64 \\
\lambda_{4} & =9,740.91 \\
\lambda_{7} & =31,560.55 \\
\lambda_{10} & =65,848.55\end{aligned}$ \\
\hline & B & $\begin{array}{l}(1,1) \\
(1,3) \\
(3,1) \\
(3,3) \\
(1,5) \\
(5,1)\end{array}$ & $427 \cdot 57$ & $\begin{array}{r}401 \cdot 61 \\
7,103 \cdot 64 \\
7,897 \cdot 60 \\
16,607 \cdot 51\end{array}$ & $\begin{array}{r}392 \cdot 04 \\
9,364 \cdot 95 \\
9,628 \cdot 80 \\
28,311 \cdot 58 \\
57,563 \cdot 00 \\
59,912 \cdot 48\end{array}$ & $\begin{array}{r}390 \cdot 14 \\
9654 \cdot 52 \\
9731 \cdot 92 \\
30,727 \cdot 04 \\
64,641 \cdot 36 \\
65,264 \cdot 62\end{array}$ & $\begin{aligned} \lambda_{1} & =389.64 \\
\lambda_{4} & =9,740 \cdot 91 \\
\lambda_{7} & =31,560 \cdot 55 \\
\lambda_{10} & =65,848 \cdot 55\end{aligned}$ \\
\hline \multirow[t]{2}{*}{ HSM } & A & $\begin{array}{l}(1,1) \\
(1,3) \\
(3,1) \\
(3,3) \\
(1,5) \\
(5,1)\end{array}$ & $305 \cdot 02$ & $\begin{array}{r}363 \cdot 03 \\
9,068 \cdot 55 \\
13,410 \cdot 28 \\
32,734 \cdot 87\end{array}$ & $\begin{array}{r}382 \cdot 31 \\
9,639 \cdot 09 \\
10,365 \cdot 19 \\
31,864 \cdot 81 \\
72,426 \cdot 86 \\
77,858 \cdot 36\end{array}$ & $\begin{array}{r}387 \cdot 64 \\
9,698 \cdot 60 \\
9,863 \cdot 15 \\
31,568 \cdot 50 \\
67,113 \cdot 19 \\
68,057 \cdot 07\end{array}$ & $\begin{aligned} \lambda_{1} & =389.64 \\
\lambda_{4} & =9,740.91 \\
\lambda_{7} & =31,560 \cdot 55 \\
\lambda_{10} & =65,848.55\end{aligned}$ \\
\hline & B & $\begin{array}{l}(1,1) \\
(1,3) \\
(3,1) \\
(3,3) \\
(1,5) \\
(5,1)\end{array}$ & $628 \cdot 33$ & $\begin{array}{r}431 \cdot 73 \\
9,415 \cdot 73 \\
13,717 \cdot 77 \\
39,898 \cdot 67\end{array}$ & $\begin{array}{r}399 \cdot 34 \\
9,922 \cdot 40 \\
10,675 \cdot 68 \\
33,273 \cdot 24 \\
73,828 \cdot 85 \\
78,406 \cdot 69\end{array}$ & $\begin{array}{r}392 \cdot 04 \\
9,783 \cdot 68 \\
9,948 \cdot 52 \\
31,917 \cdot 22 \\
67,628 \cdot 48 \\
68,494 \cdot 33\end{array}$ & $\begin{aligned} \lambda_{1} & =389 \cdot 64 \\
\lambda_{4} & =9,740 \cdot 91 \\
\lambda_{7} & =31,560.55 \\
\lambda_{10} & =65,848 \cdot 55\end{aligned}$ \\
\hline
\end{tabular}

$+\lambda=\rho \omega^{2}\left[(2 a)^{4}\right] / D$.

the expression $\lambda=\left(m^{2}+n^{2}\right)^{2} \pi^{4}$. For the clamped plate the reference solution is taken from Reference 50.

A comparison of the results for the first eigenvalue using different models is given in Table III. The calculated values using the DKT and HSM models (meshes $A$ and $B$ and a lumped diagonal mass matrix) are compared with those reported in Reference 19 using the DKT and the HCT elements with the same meshes but using a consistent mass matrix. The convergence characteristics of the DKT and HSM elements (with a lumped diagonal matrix) are compared in Figure 18 for both types of boundary conditions and meshes.

Considering the analysis results, we make the following observations.

(1) For the simply-supported plate, modes $(1,3)$ and $(3,1)$ (or $(1,5)$ and $(5,1))$ (see Table I) are theoretically associated with the same eigenvalue. The finite element results are different for these modes, but the differences between the values decrease as the mesh is refined and in every case both values converge to the reference value. (The nodal line of one of the first two modes is circular and the other nodal line is along the diagonal of the plate.) Similar observations also hold for the clamped plate and have been discussed previously using other finite element formulations. ${ }^{50,28}$ 
Table II. Free vibration frequencies of clamped square plate

\begin{tabular}{|c|c|c|c|c|c|c|c|}
\hline Element & $\begin{array}{l}\text { Mesh } \\
\text { orien- } \\
\text { tation }\end{array}$ & Mode & 1 & 2 & 4 & 8 & $\lambda \dagger$ \\
\hline \multirow[t]{2}{*}{ DKT } & A & $\begin{array}{l}(1,1) \\
(1,3) \\
(3,1) \\
(3,3) \\
(1,5) \\
(5,1)\end{array}$ & $529 \cdot 08 \ddagger$ & $\begin{array}{r}996 \cdot 29 \\
8,853 \cdot 77 \\
9,388 \cdot 27 \\
16,437 \cdot 16\end{array}$ & $\begin{array}{r}1,203 \cdot 25 \\
15,632 \cdot 67 \\
15,974 \cdot 98 \\
38,973 \cdot 41 \\
75,470 \cdot 81 \\
77,394 \cdot 62\end{array}$ & $\begin{array}{r}1,270 \cdot 82 \\
16,905 \cdot 93 \\
17,103 \cdot 20 \\
45,885 \cdot 33 \\
92,043 \cdot 40 \\
93,009 \cdot 73\end{array}$ & 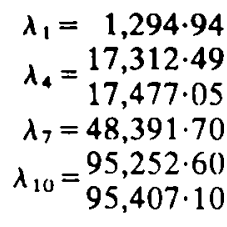 \\
\hline & B & $\begin{array}{l}(1,1) \\
(1,3) \\
(3,1) \\
(3,3) \\
(1,5) \\
(5,1)\end{array}$ & $964 \cdot 88$ & $\begin{array}{r}1,364 \cdot 60 \\
9,645 \cdot 95 \\
10,035 \cdot 82 \\
17,892 \cdot 05\end{array}$ & $\begin{array}{r}1,311 \cdot 88 \\
16,697 \cdot 68 \\
16,810 \cdot 73 \\
41,253 \cdot 31 \\
77,140 \cdot 12 \\
81,768 \cdot 01\end{array}$ & $\begin{array}{r}1,298 \cdot 94 \\
17,209 \cdot 41 \\
17,358 \cdot 68 \\
46,633 \cdot 57 \\
93,152 \cdot 85 \\
94,624 \cdot 76\end{array}$ & $\begin{aligned} \lambda_{1} & =1,294 \cdot 94 \\
\lambda_{4} & =17,312 \cdot 49 \\
17,477 \cdot 05 & \\
\lambda_{7} & =48,391 \cdot 70 \\
\lambda_{10} & =95,252 \cdot 60 \\
& 95,407 \cdot 10\end{aligned}$ \\
\hline \multirow[t]{2}{*}{ HSM } & A & $\begin{array}{l}(1,1) \\
(1,3) \\
(3,1) \\
(3,3) \\
(1,5) \\
(5,1)\end{array}$ & $1,151 \cdot 86$ & $\begin{array}{r}1,211 \cdot 13 \\
16,984 \cdot 70 \\
20,762 \cdot 39 \\
49,216 \cdot 58\end{array}$ & $\begin{array}{r}1,270 \cdot 28 \\
18,269 \cdot 91 \\
19,055 \cdot 18 \\
51,216 \cdot 57 \\
111,518 \cdot 97 \\
123,653 \cdot 50\end{array}$ & $\begin{array}{r}1,287 \cdot 59 \\
17,621 \cdot 45 \\
17,641 \cdot 53 \\
49,015 \cdot 43 \\
99,056 \cdot 45 \\
100,681 \cdot 87\end{array}$ & $\begin{array}{rl}\lambda_{1} & =1,294 \cdot 94 \\
\lambda_{4} & =17,312 \cdot 49 \\
17,477 \cdot 05 & 19 \\
\lambda_{7} & =48,391 \cdot 70 \\
\lambda_{10} & =95,252 \cdot 60 \\
95,407 \cdot 10\end{array}$ \\
\hline & B & $\begin{array}{l}(1,1) \\
(1,3) \\
(3,1) \\
(3,3) \\
(1,5) \\
(5,1)\end{array}$ & $3,455 \cdot 06$ & $\begin{array}{r}1,772 \cdot 97 \\
18,516 \cdot 59 \\
21,955 \cdot 02 \\
57,521 \cdot 09\end{array}$ & $\begin{array}{r}1,391 \cdot 51 \\
19,176 \cdot 02 \\
20,329 \cdot 77 \\
55,178 \cdot 41 \\
115,520 \cdot 79 \\
127,138 \cdot 90\end{array}$ & $\begin{array}{r}1,317 \cdot 90 \\
17,728 \cdot 86 \\
18,079 \cdot 46 \\
49,875 \cdot 53 \\
100,240 \cdot 24 \\
102,157 \cdot 34\end{array}$ & $\begin{aligned} & \lambda_{1}=1,294 \cdot 94 \\
& \lambda_{4}=17,312 \cdot 49 \\
& \lambda_{7}=48,477 \cdot 05 \\
& \lambda_{10}=95,252 \cdot 60 \\
& 95,407 \cdot 10\end{aligned}$ \\
\hline
\end{tabular}

+ Average of the upper and lower bounds in Reference 50.

$\ddagger \lambda=\rho \omega^{2}\left[(2 a)^{4}\right] / D$.

(2) The convergence rate for the first eigenvalue is similar using the DKT and HSM elements. Mesh B is more efficient for the DKT element, whereas mesh A yields better results using the HSM element. A consistent mass representation as used in Reference 19 (Table III) gives a better convergence rate than the lumped mass approach used here.

4.2.5. Free vibration analysis of a triangular cantilever. This protlem deals with the vibration of a cantilevered triangular plate representing a fin used in missile stabilization. The problem is chosen to compare the natural frequencies and mode shapes predicted by the DKT and HSM elements with experimental results and with results obtained by employing other higher order finite elements.

The data and the finite element meshes used in the analysis are given in Figure 19. The natural frequencies obtained with the DKT and HSM elements with 10 and 21 mass dof are given in Table IV. Included in the same table are experimental values and results using other finite elements. ${ }^{7,50}$ The nodal lines corresponding to the first six modes for the DKT element with 21 
Table III. Lowest eigenvalue of square plate-influence of model and mesh orientation

\begin{tabular}{|c|c|c|c|c|c|c|c|c|}
\hline \multirow[b]{3}{*}{$N$} & \multicolumn{8}{|c|}{ Simply supported square plate } \\
\hline & \multicolumn{2}{|c|}{ DKT +} & \multicolumn{2}{|c|}{$\mathrm{HSM}^{+}$} & \multicolumn{2}{|c|}{ DKT $\ddagger$} & \multicolumn{2}{|c|}{ HCT $\ddagger$} \\
\hline & $\begin{array}{c}\text { Mesh } \\
\text { A }\end{array}$ & $\begin{array}{c}\text { Mesh } \\
\text { B }\end{array}$ & $\begin{array}{c}\text { Mesh } \\
\text { A }\end{array}$ & $\begin{array}{c}\text { Mesh } \\
\text { B }\end{array}$ & $\begin{array}{c}\text { Mesh } \\
\text { A }\end{array}$ & $\begin{array}{c}\text { Mesh } \\
\text { B }\end{array}$ & $\begin{array}{c}\text { Mesh } \\
\text { A }\end{array}$ & $\begin{array}{c}\text { Mesh } \\
\text { B }\end{array}$ \\
\hline 1 & $240 \cdot 2$ & $427 \cdot 6$ & $305 \cdot 0$ & $628 \cdot 3$ & $334 \cdot 6$ & $380 \cdot 7$ & $497 \cdot 0$ & $460 \cdot 6$ \\
\hline 2 & $344 \cdot 5$ & $401 \cdot 6$ & $363 \cdot 0$ & 431.7 & $374 \cdot 8$ & $374 \cdot 1$ & $412 \cdot 1$ & $406 \cdot 1$ \\
\hline 4 & $377 \cdot 3$ & $392 \cdot 0$ & $382 \cdot 3$ & $399 \cdot 3$ & $385 \cdot 4$ & $384 \cdot 6$ & $395 \cdot 0$ & $393 \cdot 7$ \\
\hline 8 & $386 \cdot 5$ & $390 \cdot 1$ & 387.6 & $392 \cdot 0$ & & & & \\
\hline
\end{tabular}

Reference value: $\quad 389.6$

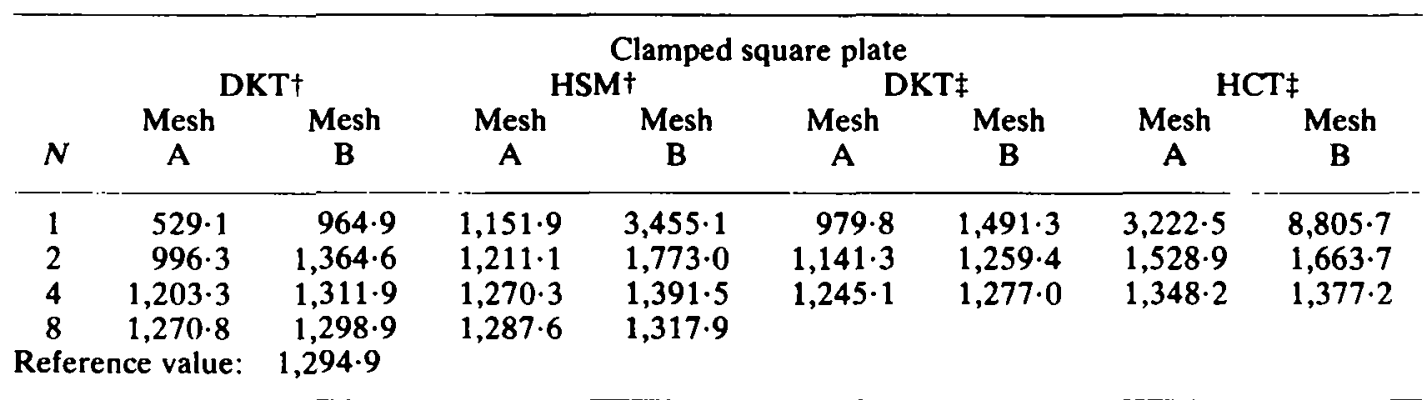

† Lumped mass.

$\ddagger$ Consistent mass. ${ }^{19}$

Table IV. Natural frequencies of cantilevered triangular plate

\begin{tabular}{|c|c|c|c|c|c|c|c|}
\hline & \multicolumn{2}{|c|}{ DKT } & \multicolumn{2}{|c|}{ HSM } & \multirow{2}{*}{$\begin{array}{c}\text { Shell } \\
\text { element } \\
N=4 \\
36 \text { mass } \\
\text { dof } \\
\text { (lumped } \\
\text { mass) }\end{array}$} & \multirow{2}{*}{$\begin{array}{c}\text { High } \\
\text { precision } \\
\text { element }^{\text {so }} \\
\\
\begin{array}{c}N=3 \\
40 \text { mass } \\
\text { dof }\end{array}\end{array}$} & \multirow[t]{2}{*}{$\begin{array}{l}\text { Experi- } \\
\text { mental } \\
\text { value }^{50}\end{array}$} \\
\hline Mode & $\begin{array}{c}N=4 \\
10 \text { mass } \\
\text { dof }\end{array}$ & $\begin{array}{c}N=6 \\
21 \text { mass } \\
\text { dof }\end{array}$ & $\begin{array}{c}N=4 \\
10 \text { mass } \\
\text { dof }\end{array}$ & $\begin{array}{c}N=6 \\
21 \text { mass } \\
\text { dof }\end{array}$ & & & \\
\hline 1 & $34 \cdot 5+$ & $35 \cdot 7$ & $34 \cdot 8$ & $35 \cdot 8$ & $36 \cdot 7$ & $36 \cdot 6$ & $34 \cdot 5$ \\
\hline 2 & $117 \cdot 6$ & $128 \cdot 6$ & $121 \cdot 3$ & $130 \cdot 7$ & $133 \cdot 5$ & $139 \cdot 3$ & 136 \\
\hline 3 & $155 \cdot 6$ & $174 \cdot 8$ & $164 \cdot 1$ & $179 \cdot 1$ & $177 \cdot 8$ & $194 \cdot 1$ & 190 \\
\hline 4 & $271 \cdot 1$ & $299 \cdot 6$ & 288.6 & $308 \cdot 2$ & $323 \cdot 0$ & $333 \cdot 8$ & 325 \\
\hline 5 & $331 \cdot 2$ & 395.6 & $365 \cdot 2$ & $413 \cdot 1$ & $410 \cdot 0$ & 455.4 & 441 \\
\hline 6 & $403 \cdot 7$ & $487 \cdot 6$ & $461 \cdot 9$ & 518.8 & $517 \cdot 0$ & 593.2 & 578 \\
\hline 7 & 474.9 & $571 \cdot 7$ & 556.4 & $615 \cdot 0$ & $621 \cdot 1$ & $671 \cdot 3$ & \\
\hline 8 & $513 \cdot 1$ & $661 \cdot 9$ & $665 \cdot 8$ & $717 \cdot 2$ & 753.4 & $811 \cdot 6$ & \\
\hline 9 & 567.0 & $744 \cdot 9$ & 703.6 & 814.9 & 997.6 & $969 \cdot 6$ & \\
\hline 10 & $667 \cdot 8$ & $868 \cdot 9$ & 935.0 & $991 \cdot 8$ & $1179 \cdot 0$ & $1126 \cdot 6$ & \\
\hline
\end{tabular}

$\uparrow$ Frequency in $\mathbf{H z}$. 

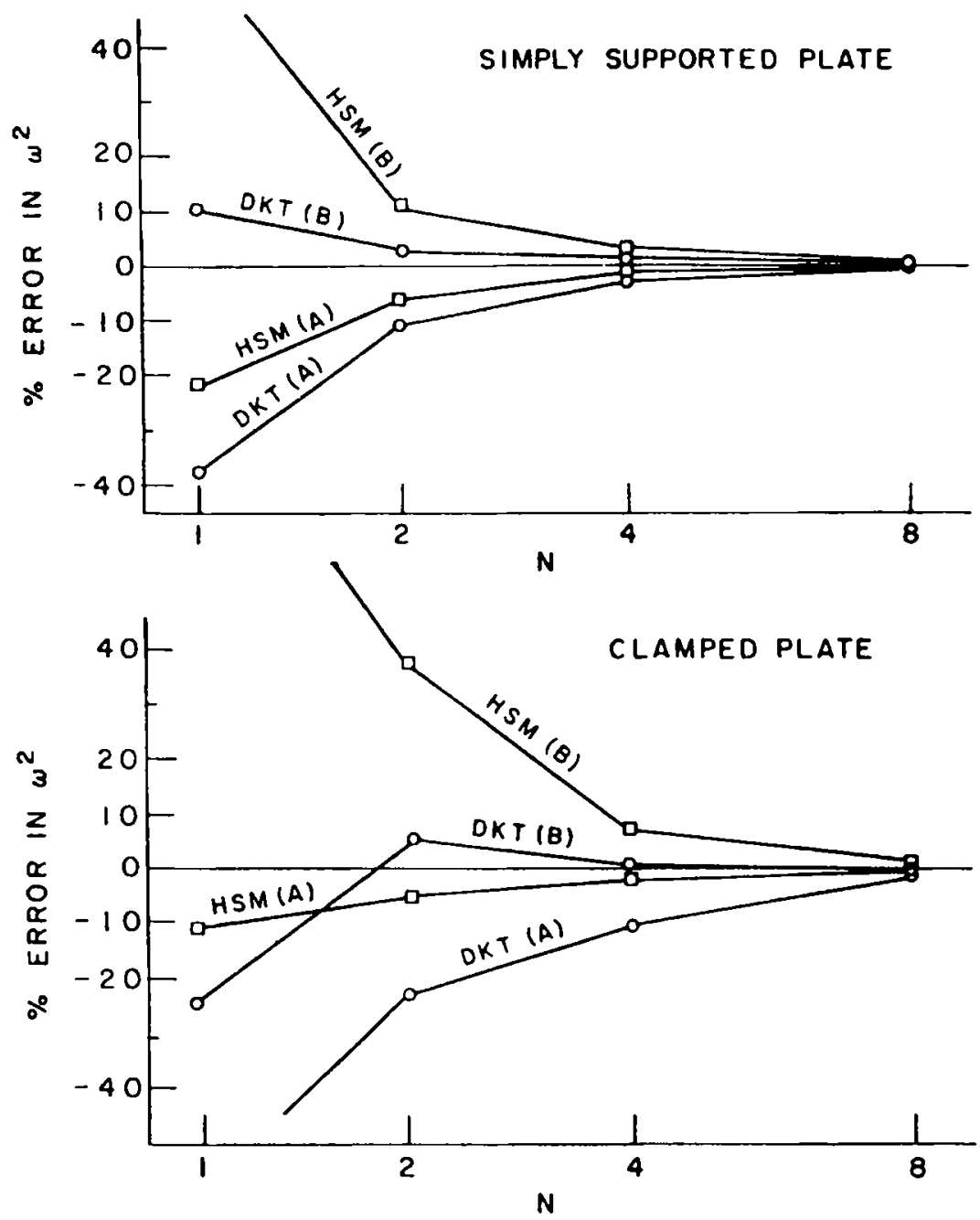

Figure 18. Error of the first eigenvalue of a square plate vs. mesh and model

mass dof are illustrated in Figure 20, together with experimental results. Considering the results we make the following observations.

(1) The DKT and HSM elements predict the fundamental frequency accurately. However, the higher frequencies are calculated less accurately.

(2) The 21 mass dof models predict the higher frequencies more accurately than the 10 mass dof ones. However, considering the fundamental frequency, the larger dof models give values that are further away from the experimental result. This numerical prediction can be explained by the fact that the finite element solution converges to the Kirchhoff plate theory result, which deviates from the experimental one due to assumptions and experimental errors.

(3) The first three mode shapes are represented accurately by the DKT element. Qualitative representation is still retained in the higher modes. 


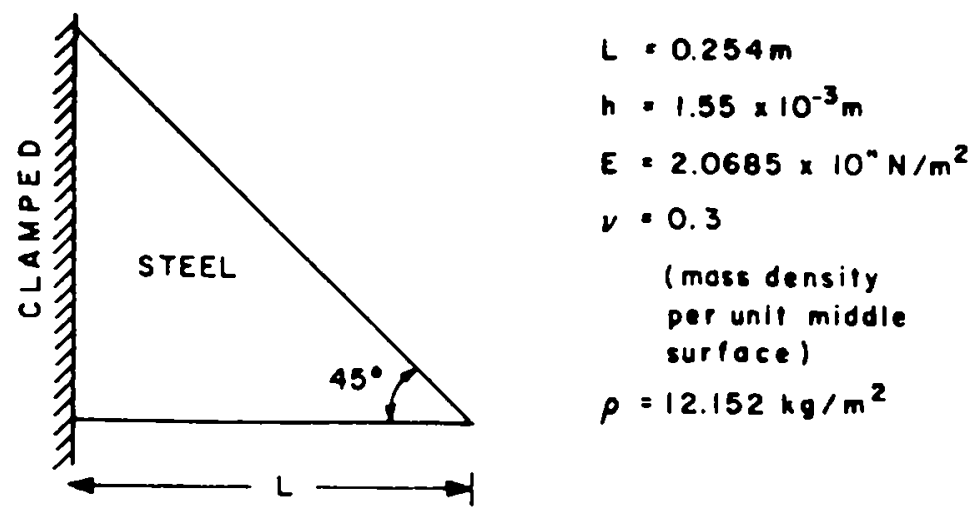

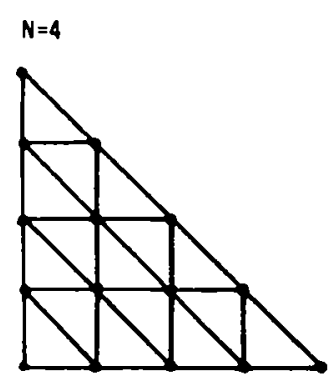

MESHES FOR DKT \& HSM ELEMENTS

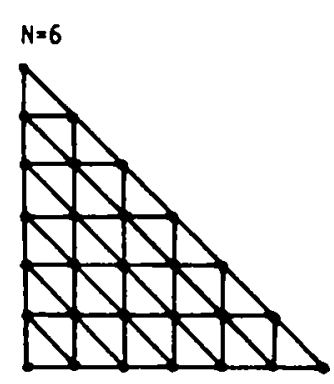

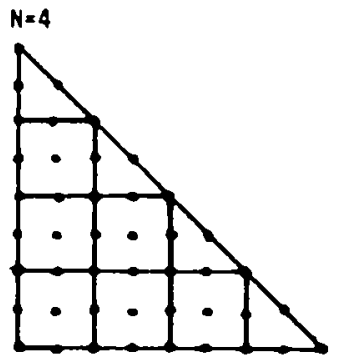

MESH FOR SHELL ELEHENT [?]

Figure 19. Triangular cantilevered plate with finite element meshes used in the analysis

\subsection{Results of some sample static problems using the SRI element}

The standard square isotropic plate $\left(\nu=0.3, k=\frac{5}{6}\right)$ was analysed to test the behaviour of the simple triangular element based on the reduced integration technique. The concentrated load condition was considered and mesh $\mathrm{A}(N=1,2,4,8,12)$ was used (Figure 5) for the clamped plate and the simply-supported plate. The solution results are given in Tables $\mathrm{V}$ and VI for different values of side to thickness ratios $(2 a / h)$. These results show that considering a thick plate the element converges to the thick plate solution (see, for example, Reference 60); however, the element cannot be employed satisfactorily for the analysis of thin plates.

The selective or reduced integration technique when applied to two-node beam and fournode quadrilateral elements gives good results in the analysis of some problems of thin beams and plates for aspect ratios up to $10^{4}$ (References 42 and 57). However, considering the present 


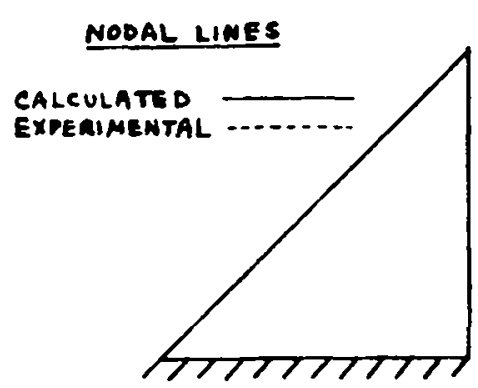

$\lambda_{1}$

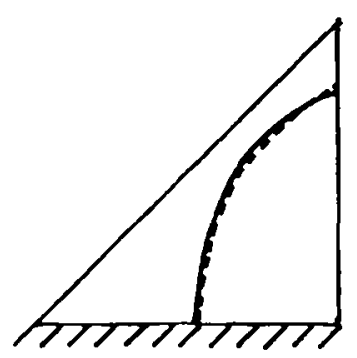

$\lambda_{3}$

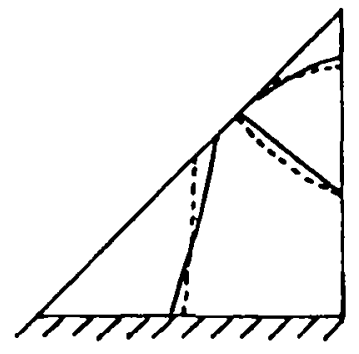

$\lambda_{s}$

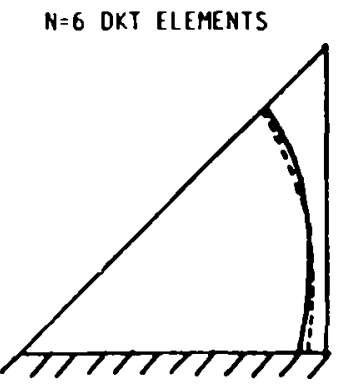

$\lambda_{2}$

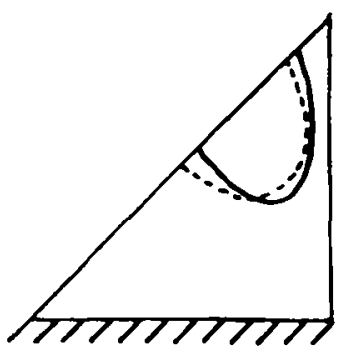

$\lambda_{4}$

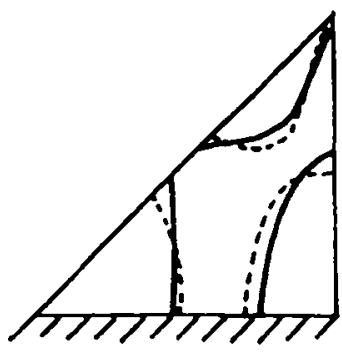

$\lambda_{6}$

Figure 20. Nodal lines for the triangular cantilevered plate

triangular element, the behaviour of the SRI element was not found acceptable for the two standard tests; hence, no other problems were considered using this element.

\section{CONCLUSIONS}

In this study, the discrete Kirchhoft theory (DKT) and hybrid stress model (HSM) elements have been found to be the most effective 9 dof triangular elements available for bending analysis of thin plates. Both elements can be formulated and implemented very efficiently. Their effectiveness has been observed in a variety of static and dynamic analyses. Based on theoretical and computational considerations and the numerical results, the DKT element is found to be somewhat superior to the HSM element. Because of little storage requirements, these two elements appear to be very promising for structural analysis on mini- and micro-computers. A 
Table V. Central deflection of a clamped plate subjected to a concentrated centre-point load (SRI elements, mesh A)

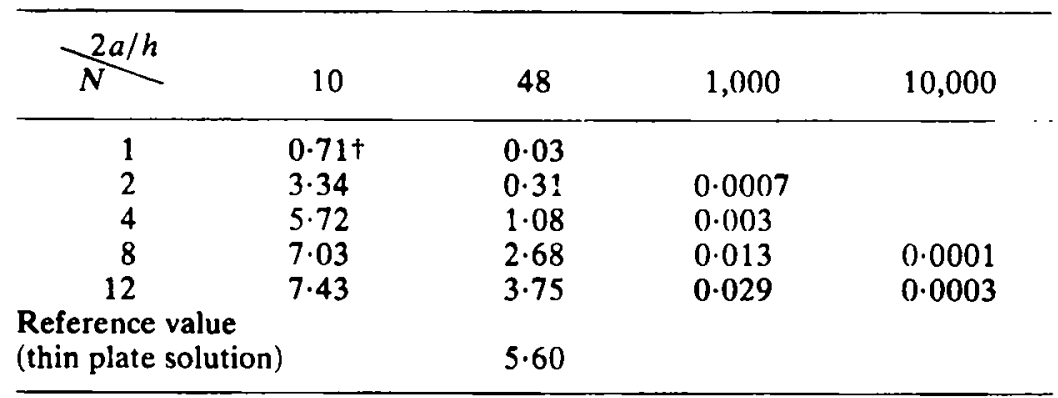

$+w_{c} D /\left\{P(2 a)^{2}\right\} \times 10^{-3} \quad k=\frac{5}{6} \quad \nu=0 \cdot 3$

Table VI. Central deflection of a simply-supported plate subjected to a concentrated centre-point load (SRI elements, mesh A)

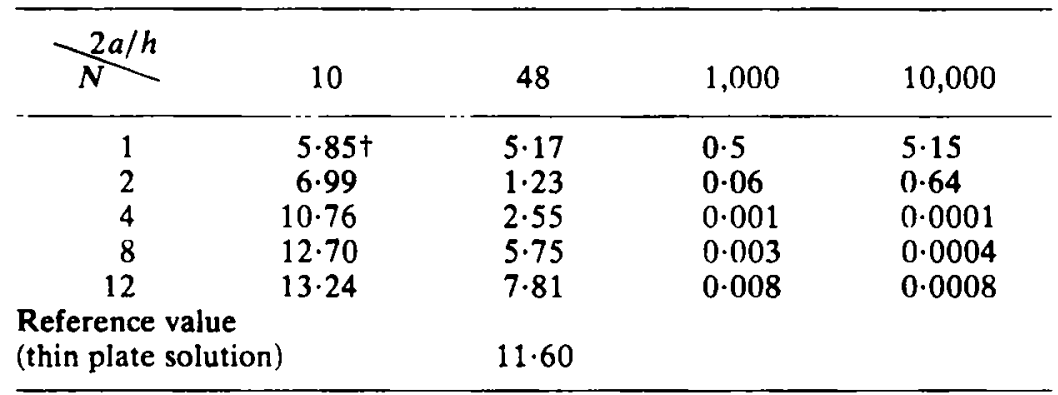

simple triangular element formulated using the theory of plates with transverse shear included and using selective integration was found to be not as effective.

\section{ACKNOWLEDGEMENTS}

We gratefully acknowledge the financial support of the ADINA users group and thank Prof. G. Dhatt, Université Laval, Quebec, Canada for helpful discussions on the DKT element.

\section{APPENDIX A: EXPANSIONS FOR THE DKT ELEMENT}

Shape function for DKT element

$$
\begin{aligned}
& N_{1}=2(1-\xi-\eta)\left(\frac{1}{2}-\xi-\eta\right) \\
& N_{2}=\xi(2 \xi-1) \\
& N_{3}=\eta(2 \eta-1) \\
& N_{4}=4 \xi \eta \\
& N_{5}=4 \eta(1-\xi-\eta) \\
& N_{6}=4 \xi(1-\xi-\eta)
\end{aligned}
$$

$\xi$ and $\eta$ are the area co-ordinates $L_{2}$ and $L_{3}$ (Reference 10) (Figure 21). 


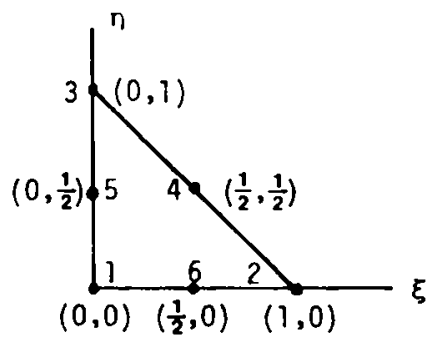

Figure 21. Co-ordinates $\xi$ and $\eta$

The derivatives of the $\mathbf{H}_{x}$ and $\boldsymbol{H}_{\boldsymbol{y}}$ functions with respect to $\xi$ and $\eta$ are obtained using equation (27). Since. the functions $N_{i}$ are not entirely symmetric in terms of $\xi$ and $\eta$, the following arrangement is proposed:

$$
\mathbf{H}_{x, \xi}=\left[\begin{array}{l}
P_{6}(1-2 \xi)+\left(P_{5}-P_{6}\right) \eta \\
q_{6}(1-2 \xi)-\left(q_{5}+q_{6}\right) \eta \\
-4+6(\xi+\eta)+r_{6}(1-2 \xi)-\eta\left(r_{5}+r_{6}\right) \\
-P_{6}(1-2 \xi)+\eta\left(P_{4}+P_{6}\right) \\
q_{6}(1-2 \xi)-\eta\left(q_{6}-q_{4}\right) \\
-2+6 \xi+r_{6}(1-2 \xi)+\eta\left(r_{4}-r_{6}\right) \\
-\eta\left(P_{5}+P_{4}\right) \\
\eta\left(q_{4}-q_{5}\right) \\
-\eta\left(r_{5}-r_{4}\right)
\end{array}\right]
$$

$$
\mathbf{H}_{y, \xi}=\left[\begin{array}{l}
t_{6}(1-2 \xi)+\eta\left(t_{5}-t_{6}\right) \\
1+r_{6}(1-2 \xi)-\eta\left(r_{5}+r_{6}\right) \\
-q_{6}(1-2 \xi)+\eta\left(q_{5}+q_{6}\right) \\
-t_{6}(1-2 \xi)+\eta\left(t_{4}+t_{6}\right) \\
-1+r_{6}(1-2 \xi)+\eta\left(r_{4}-r_{6}\right) \\
-q_{6}(1-2 \xi)-\eta\left(q_{4}-q_{6}\right) \\
-\eta\left(t_{4}+t_{5}\right) \\
\eta\left(r_{4}-r_{5}\right) \\
-\eta\left(q_{4}-q_{5}\right)
\end{array}\right]
$$




$$
\mathbf{H}_{x, \eta}=\left[\begin{array}{l}
-P_{5}(1-2 \eta)-\xi\left(P_{6}-P_{5}\right) \\
q_{5}(1-2 \eta)-\xi\left(q_{5}+q_{6}\right) \\
-4+6(\xi+\eta)+r_{5}(1-2 \eta)-\xi\left(r_{5}+r_{6}\right) \\
\xi\left(P_{4}+P_{6}\right) \\
\xi\left(q_{4}-q_{6}\right) \\
-\xi\left(r_{6}-r_{4}\right) \\
P_{5}(1-2 \eta)-\xi\left(P_{4}+P_{5}\right) \\
q_{5}(1-2 \eta)+\xi\left(q_{4}-q_{5}\right) \\
-2+6 \eta+r_{5}(1-2 \eta)+\xi\left(r_{4}-r_{5}\right) \\
\mathbf{H}_{y, \eta}=\left[\begin{array}{l}
-t_{5}(1-2 \eta)-\xi\left(t_{6}-t_{5}\right) \\
1+r_{5}(1-2 \eta)-\xi\left(r_{5}+r_{6}\right) \\
-q_{5}(1-2 \eta)+\xi\left(q_{5}+q_{6}\right) \\
\xi\left(t_{4}+t_{6}\right) \\
\xi\left(r_{4}-r_{6}\right) \\
-\xi\left(q_{4}-q_{6}\right) \\
t_{5}(1-2 \eta)-\xi\left(t_{4}+t_{5}\right) \\
-1+r_{5}(1-2 \eta)+\xi\left(r_{4}-r_{5}\right) \\
-q_{5}(1-2 \eta)-\xi\left(q_{4}-q_{5}\right)
\end{array}\right]
\end{array}\right.
$$

where

$$
\begin{array}{rlrl}
P_{k} & =-6 x_{i j} / l_{i j}^{2}=6 a_{K} ; & t_{k}=-6 y_{i j} / l_{i j}^{2}=6 d_{K} \\
q_{k}=3 x_{i j} y_{i j} / l_{i j}^{2}=4 b_{K} ; & r_{k}=3 y_{i j}^{2} / l_{i j}^{2} \\
k & =4,5,6 \quad \text { for } i j=23, & 31,12, \text { respectively. }
\end{array}
$$

Using the above expressions and equation (30) the $\mathbf{B}$ matrix needed to evaluate $\mathbf{K}_{\mathrm{DKT}}$, equation (31), and $\mathbf{M}$, equation (32), is readily obtained. 


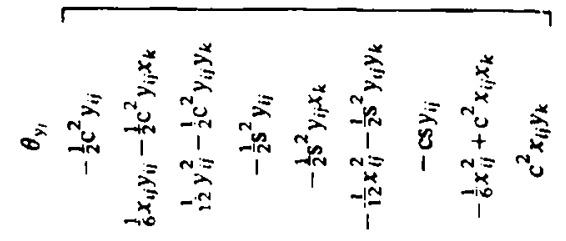

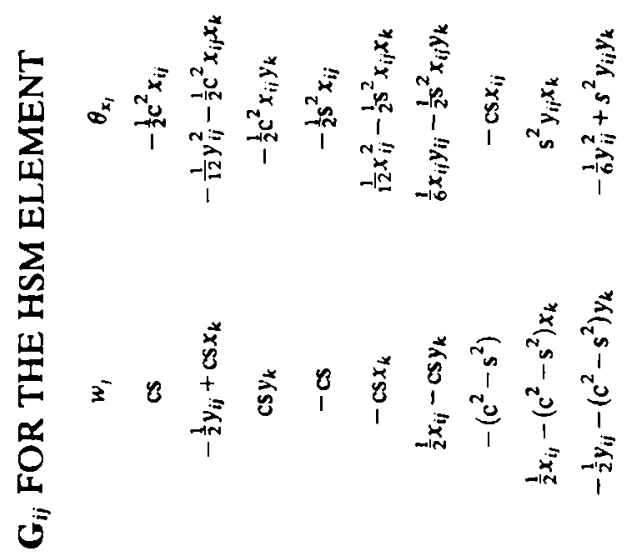

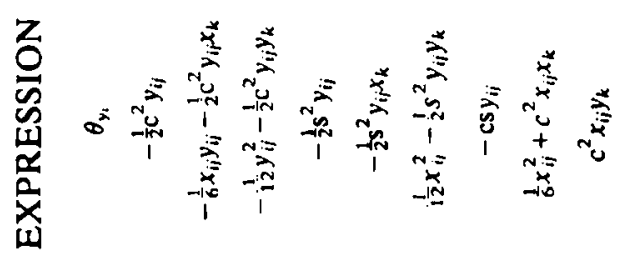

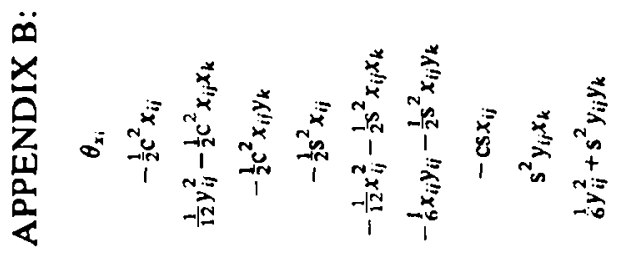

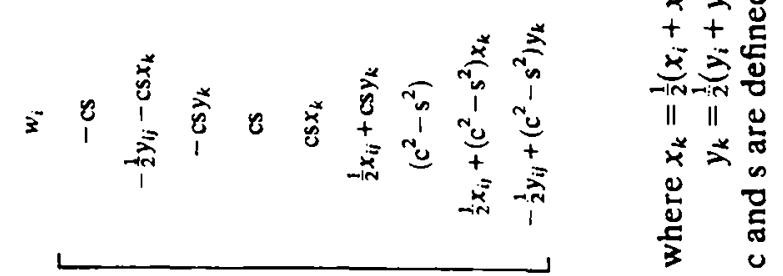




\section{APPENDIX C: EQUIVALENCE BETWEEN THE REDUCED INTEGRATION DISPLACEMENT METHOD AND A MIXED HELLINGER-REISSNER PRINCIPLE FORMULATION}

Using the Lagrange multiplier technique the total potential energy function governing plate bending with the transverse shear effects included can be written as

$$
\Pi_{\mathrm{HR}}=\sum_{m} \int_{A}\left(\boldsymbol{\kappa}^{\mathrm{T}} \mathbf{D}_{b} \boldsymbol{\kappa}-\frac{1}{2} \boldsymbol{K}^{* \mathrm{~T}} \mathbf{D}_{b} \boldsymbol{K}^{*}+\boldsymbol{\gamma}^{\mathrm{T}} \mathbf{D}_{s} \boldsymbol{\gamma}^{*}-\frac{1}{2} \boldsymbol{\gamma}^{* \mathrm{~T}} \mathbf{D}_{s} \boldsymbol{\gamma}^{*}\right) \mathrm{d} x \mathrm{~d} y-W^{(m)}
$$

where

$$
W^{(m)}=\int_{A} w \bar{p}_{z} \mathrm{~d} x \mathrm{~d} y+\int_{\mathrm{C}_{\sigma}}\left(w \bar{V}_{z}+\beta_{\mathrm{x}} \bar{M}_{\mathrm{x} n}+\beta_{y} \bar{M}_{\mathrm{yn}}\right) \mathrm{d} s
$$

$\kappa, \gamma, D_{b}, D_{s}$ are given by equations (3), (4), (10) and (11), respectively, and

$$
\kappa^{* T}=\left[\begin{array}{lll}
\kappa_{x}^{*} & \kappa_{y}^{*} & \kappa_{x y}^{*}
\end{array}\right] \text { and } \gamma^{* T}=\left[\begin{array}{ll}
\gamma_{x}^{*} & \gamma_{y}^{*}
\end{array}\right]
$$

are independent strain components that are not related to the displacements $w, \beta_{x}$ and $\beta_{y}$. Also $\bar{P}_{z}$ is a uniform normal load in the $z$ direction, $C_{\sigma}$ is the part of the boundary where $\bar{V}_{z}$ (shear force), and $\bar{M}_{x n}, \bar{M}_{y n}$ (moments) are prescribed.

The independent quantities subject to variation in equation (76) are $\kappa^{*}, \gamma^{*}, w, \beta_{x}$ and $\beta_{y}$ with the subsidiary conditions

$$
w=\bar{W} \quad \beta_{x}=\bar{\beta}_{x} \text { and } \beta_{y}=\bar{\beta}_{y} \text { on } C_{u}
$$

Equation (76) can be considered as the Hellinger-Reissner functional in the theory of plates with transverse shear included. If the curvature-displacement relations are satisfied, i.e.

$$
\mathbf{K}=\mathbf{k}^{*}
$$

a modified functional is obtained:

$$
\Pi_{\mathrm{HR}}^{\mathrm{mod}}=\sum_{m} \int_{A}\left(\frac{1}{2} \mathbf{K}^{\mathrm{T}} \mathbf{D}_{b} \mathbf{k}+\boldsymbol{\gamma}^{\mathrm{T}} \mathbf{D}_{s} \boldsymbol{\gamma}^{*}-\frac{1}{2} \boldsymbol{\gamma}^{* \mathrm{~T}} \mathbf{D}_{s} \boldsymbol{\gamma}^{*}\right) \mathrm{d} x \mathrm{~d} y-W^{(m)}
$$

The independent quantities in equation (80) are $w, \beta_{x}, \beta_{y}$ and $\boldsymbol{\gamma}^{*}$ with equation (78) as subsidiary conditions.

Finite elements can be formulated based on equation (80), with $w, \beta_{x}$ and $\beta_{y}$ as nodal dof and $\gamma^{*}$ being expressed in terms of unknown generalized parameters $\alpha_{i}$ defined on the individual elements.

If we consider a triangular element with $w, \beta_{x}$ and $\beta_{y}$ as given by equation (69) and $\gamma^{*}$ a vector of two constant parameters:

$$
\boldsymbol{\gamma}^{*}=\left[\begin{array}{l}
\alpha_{1} \\
\alpha_{2}
\end{array}\right]=\boldsymbol{\alpha}
$$

then using equations (80), (81) and (23), (69) we have:

$$
\Pi_{\mathbf{H R}}^{\bmod }=\sum_{m} \frac{1}{2} \mathbf{U}^{\mathrm{T}} \mathbf{K}_{b} \mathbf{U}+\boldsymbol{\alpha}^{\mathrm{T}} \mathbf{G}_{\boldsymbol{\alpha}} \mathbf{U}-\frac{1}{2} \boldsymbol{\alpha}^{\mathrm{T}} \mathbf{H}_{\boldsymbol{\alpha}} \boldsymbol{\alpha}-\mathbf{U}^{\mathrm{T}} \mathbf{Q}
$$

where $K_{b}$ is given, by equation (71)

$$
\mathbf{G}_{\alpha}=D_{s} \int_{A} B_{s} \mathrm{~d} x \mathrm{~d} y
$$


and

$$
\mathbf{H}_{\alpha}=A \mathbf{D}_{\text {s }}
$$

The vector $\mathbf{Q}$ is the element equivalent nodal-point forces corresponding to $W^{(m)}$ equation (76). The stationary conditions associated with (82) give the following relations for each element:

$$
\mathbf{G}_{\boldsymbol{\alpha}} \mathbf{U}-\mathbf{H}_{\boldsymbol{\alpha}} \boldsymbol{\alpha}=0
$$

or

$$
\boldsymbol{\alpha}=\mathbf{H}^{-1} \mathbf{G}_{\alpha} \mathbf{U}
$$

Using equation (84) in equation (82) gives

$$
\Pi_{\mathbf{H R}}^{\bmod }=\sum_{m} \frac{1}{2} \mathbf{U}^{\mathrm{T}} \mathbf{K}_{\mathbf{H R}}^{\bmod } \mathbf{U}-\mathbf{U}^{\mathrm{T}} \mathbf{Q}
$$

where

$$
\mathbf{K}_{\mathbf{H R}}^{\bmod }=\mathbf{K}_{b}+\boldsymbol{K}_{s}^{*}
$$

with

$$
\mathbf{K}_{\mathbf{s}}^{*}=\mathbf{G}_{\alpha}^{\mathrm{T}} \mathbf{H}_{\alpha}^{-1} \mathbf{G}_{\alpha}
$$

Using equation (83) we have

$$
\mathbf{K}_{s}^{*}=\frac{1}{A} \mathbf{R}_{s}^{\mathrm{T}} \mathbf{D}_{s} \mathbf{R}_{s}
$$

with

$$
\mathbf{R}_{s}=\int_{A} \mathbf{B}_{s} \mathrm{~d} A
$$

Thus the matrix $\mathbf{K}_{s}^{*}$ corresponds to the matrix $\mathbf{K}_{s}$ defined in equation (73) using the reduced integration (one-point) scheme. The above derivation shows the equivalence between the two different formulations and gives the variational principle associated with the reduced integration displacement formulation for the present triangular element.

\section{REFERENCES}

1. R. H. Gallagher, 'Geometrically nonlinear shell analysis’, Proc. Int Conf. in Nonlinear Solid and Structural Mech., Col-1/Col-26, Geilo, Norway, 1977.

2. R. H. Gallagher, 'Shell elements', Proc., World Congr. on F.E.M., in Struct. Mech., vol. 1, Bournemouth, England, 1975.

3. D. Ashwell and R. H. Gallagher, Finite Elements for Thin Shells and Curved Members, Wiley, London, 1976.

4. D. Bushnell, 'Thin shells', Proc. Symp. Struct. Mech. Computer Programs (Eds, Pilkey et al.), Univ. Press of Virginia, Charlottesville, 1974, pp. 277-358.

5. J. L. Batoz, 'Analyse non linéaire des coques minces élastiques de formes arbitraires par éléments triangulaires courbés', $D$. Sc. thesis, Univ. Laval, Quebec, Canada, 1977.

6. E. Ramm, 'A plate/shell element for large deflections and rotations', in Formulations and Computation Algorithms in Finite Element Analysis (Eds Bathe, K. J., Oden, J. T. and Wunderlich, W.), MIT Press, 1976.

7. K. J. Bathe and S. Bolourchi, 'A geometric and material nonlinear plate and shell element', J. Comp. Struct., $11,23-40$ (1980).

8. J. H. Argyris and P. C. Dunne, 'Nonlinear and post-buckling analysis of structures', in Formulations and Computation Algorithms in Finite Element Analysis (Eds Bathe, K. J. et al.), MIT Press, 1976. 
9. G. Horrigmoe, 'Finite element instability analysis of free-form shells', Report No. 77-2, Norwegian Institute of Technology, Univ, of Trondheim, Norway, 1977.

10. O. C. Zienkiewicz, The Finite Element Method, 3rd edn, McGraw-Hill, London, 1977.

11. K. J. Bathe and E. L. Wilson, Numerical Methods in Finite Element Analysis, Prentice-Hall, Englewood Cliffs, N.J., 1976.

12. Y. Yoshida, 'A hybrid stress element for thin shell analysis', Proc. Conf. on Finite Element Methods in Engineering, Univ. of South Wales, 1974.

13. M. D. Olson and T. W. Bearden, 'The simple flat triangular shell element revisited', Int. J. num. Meth. Engng, 14(1), $51-68$ (1979).

14. G. Horrigmoe, 'Hybrid stress finite element model for nonlinear shell problems', Proc. 6th Canadian Conf. on Applied Mech., U.B.C. Vancouver, Canada, 1977.

15. J. H. Argyris, P. C. Dunne, G. A. Malejannakis and E. Schelkie, 'A simple triangular facet shell element with applications to linear and nonlinear equilibrium and elastic stability problems', Comp. Meth. Appl. Mech. Eng. 10, $371-403$ (1977).

16. R. W. Clough and J. L. Tocher, 'Finite element stiffness matrices for analysis of plate bending', Proc. Conf. on Matrix Methods in Structural Mechanics, WPAFB, Ohio, 1965, pp. 515-545.

17. G. P. Bazeley, Y. K. Cheung, B. M. Irons and O. C. Zienkiewicz, 'Triangular elements in plate bending-conforming and non-conforming solutions', Proc. Conf. on Matrix Methods in Structural Mechanics, WPAFB, Ohio, 1965, pp. 547-576.

18. R. W. Clough and C. A. Felippa, 'A refined quadrilateral element for analysis of plate bending', Proc. Conf. on Matrix Methods in Structural Mechanics, WPAFB, Ohio, 1968, pp. 399-440.

19. F. Kikuchi, 'On a finite element scheme based on the discrete Kirchhof assumption', Num. Math. 24, $211-231$ (1975).

20. C. P. Heins, Applied Plate Theory for the Engineer, Lexington Books, Mass., 1976.

21. A. Razzaque, 'Program for triangular bending elements with derivative smoothing', Int. J. num. Meth. Engng, 6, 333-343 (1973).

22. J. J. Connor and G. Will, 'A triangular flat plate bending element', Rep. TR-68-3, Dept of Civil Eng., MIT, Cambridge, Mass., 1968.

23. R. H. Gallagher, Finire Element Analysis: Fundamentals, Prentice-Hall, Englewood Cliffs, N.J., 1975.

24. T. Pian, 'Element stifiness matrices for prescribed boundary stresses', Proc. Conf. on Matrix Methods in Structural Mechanics, WPAFB, Ohio, 1965, pp. 457-477.

25. R. J. Allwood and G. M. Cornes, 'A polygonal finite element for plate bending problems using the assumed stress approach' Int. J. num. Meth. Engng, 1, 135-149 (1969).

26. D. J. Allman, 'Triangular finite elements for plate bending with constant and linearly varying bending moments', Proc. IUTAM Conf. on High Speed Computing of Elastic Structures, Liege, 1970, pp. 105-136.

27. Y. Yoshida, 'Equivalent finite elements of different bases', Proc. Advances in Comp. Methods in Structural Mechanics and Design (Eds Oden, Clough, Yamamoto), Univ. of Alabama Press, Huntsville, 1972, pp. $133-149$.

28. B. K. Neale, R. D. Henshell and G. Edwards, 'Hybrid plate bending elements', J. Sound Vibration, 23(1), 101-112 (1972).

29. P. Bartholomew, 'Comment of hybrid finite elements', Int. J. num. Meth. Engng, 10(4), 968-973 (1976).

30. T. Pian and P. Tong. 'Basis of finite element methods for solid continua', Int. J. num. Meth. Engng, 1, 3-28 (1969).

31. D. J. Allman, 'A simple cubic displacement element for plate bending', Int. J. num. Meth. Engng, 10(2), 263-281 (1976).

32. H. Mang and R. Gallagher, 'A critical assessment of the simplified hybrid displacement method', Int. J. num. Meth. Engng, 11, 145-167 (1977).

33. J. A. Stricklin, W. Haisler, P. Tisdale and R. Gunderson, 'A rapidly converging triangular plate element', $A I A A J$. 7(1), 180-181 (1969).

34. G. Dhatt, 'Numerical analysis of thin shells by curved triangular elements based on discrete Kirchhoff hypothesis', Proc. ASCE Symp. on Applications of FEM in Civil Engineering, Vanderbilt Univ., Nashville, Tenn., 1969, pp. 13-14.

35. G. Dhatt, 'An efficient triangular shell element', AIAA J. 8(11), 2100-2102 (1970).

36. G. Dhatt and S. Venkatasubbu, 'Finite element analysis of containment vessels', Proc. First Conf. on Struct. Mech. in Reactor Tech., vol 5, paper J3/6, Berlin, Germany, 1971.

37. G. Strang and G. J. Fix, An Analysis of the Finite Element Method, Prentice-Hall, Englewood Cliffs, N.J., 1973.

38. I. Fried, 'Residual energy balancing technique in the generation of plate bending finite elements', Comp. Struct. 4(4), $771-778$ (1974).

39. I. Fried and S. K. Yang, 'Triangular, nine-degrees of freedom, $C^{0}$ plate bending element of quadratic accuracy', Q. Appl. Math. 31(3), 303-312 (1973).

40. I. Fried, 'Shear in $C^{6}$ and $C^{1}$ bending finite elements', Int. J. Sol. Struct. 9(4) 449-460 (1973).

41. O. C. Zienkiewicz, R. L. Taylor and J. M. Too, 'Reduced integration technique in general analysis of plates and shell', Int. J. num. Meth. Engng, 3, 575-586 (1971).

42. T. Hughes, R. Taylor and W. Kanoknukulchai, 'A simple and efficient finite element for plate bending', Int. J. num. Meth. Engng, 11, 1529-1543 (1977). 
43. M. D. Olson, 'Compatibility', Proc. World Congr. on FEM in Struct. Mech., Bournemouth, England, 1975.

44. S. Utku, 'On derivation of stiffness matrices with $C^{0}$ rotation fields for plates and shells', Proc. 3rd Conf. on Matrix Methods in Struct. Mech., WPAFB, Ohio, 1971, pp. 255-274.

45. S. W. Lee and T. Pian, 'Improvement of plate and shell finite elements by mixed formulations', AIAA J. 16(1), 29-34 (1978).

46. K. Washizu, Variational Methods in Elasticity and Plasticity, 2nd edn, Pergamon Press, Oxford, 1975.

47. T. A. Rock and E. Hinton, 'A finite element method for free vibration of plates allowing for transverse shear deformation', Comp. Struct. 6, pp. 37-44.

48. S. Timoshenko and S. Woinowsky-Krieger, Theory of Plates and Shells, 2nd edn, McGraw-Hill, New York, 1969.

49. K. J. Bathe, 'ADINA-a finite element program for automatic dynamic incremental nonlinear analysis', Acoustics and Vibration Lab., Rep. 82448-1, Dept of Mech. Eng., MIT, 1975 (revised 1978).

50. G. R. Cowper, E. Kosko, G. M. Lindberg and M. D. Olson, 'Static and dynamic application of high precision triangular plate bending element', AIAA J. 7(10), 1957-1965 (1969).

51. R. D. Cook, 'Two hybrid elements for analysis of thick, thin and sandwich plates', Ini. J. num. Meth. Engng, 5, 227-288 (1972).

52. F. Kikuchi and Y. Ando, 'Some finite element solutions for plate bending problems by the simplified hybrid displacement method', Nucl. Eng. Des. 23, 155-178 (1972).

53. J. L. Batoz, K. J. Bathe and L. W. Ho, 'A search for the optimum three-node triangular plate bending element', Acoustics and Vibration Lab., Rep. 82448-8, Dept of Mech. Eng., MIT, 1978.

54. E. D. Pugh, E. Hinton and O. C. Zienkiewicz, 'A study of quadrilateral plate bending elements with reduced integration', Int. J. num. Meth. Engng, 12(7), 1059-1078 (1978).

55. O. C. Zienkiewicz, J. Bauer, K. Morgan and E. Onate, 'A simple and efficient element for axisymmetric shells', Int. J. num. Meth. Engng, 11(10), 1545-1558 (1977).

56. D. S. Malkus and T. J. Hughes, 'Mixed finite element methods-reduced and selective integration techniques: a unification of concepts', Comp. Meth. Appl. Mech. Engng 15(1), 63-81 (1978).

57. E. Hinton and E. D. L. Pugh, 'Some quadrilateral isoparametric finite elements based on Mindlin plate theory', Proc. Symp. on Applications of Computer Methods in Engineering, Los Angeles, Cal., 1977.

58. J. T. Oden and K. J. Bathe, 'A commentary on computational mechanics', Appl. Mech. Rev. 31(8), 1053-1058 (1978)

59. J. C. Debus, 'Réponse des voiles minces aux variables aléatoires', $D$. Sc. Thesis, Univ. Lavai, Quebec, Canada, 1972.

60. C. W. Pryor, Jr., R. M. Barker and D. Frederick, 'Finite element bending analysis of Reissner plates', J. Eng. Mech. Div., ASCE, 967-983 (1970). 